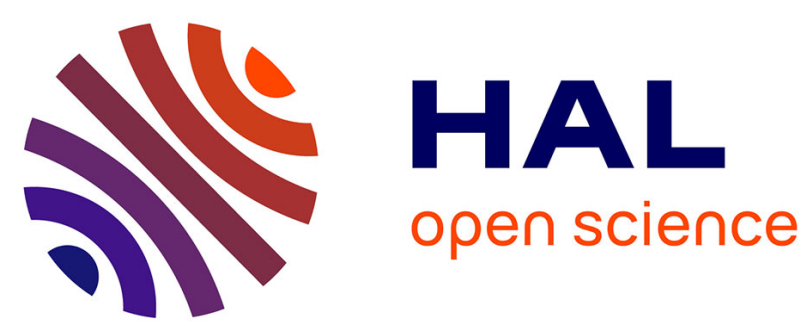

\title{
Le port de Lattara: premiers acquis sur les phases préromaines et romaines
}

Dominique Garcia

\section{To cite this version:}

Dominique Garcia. Le port de Lattara: premiers acquis sur les phases préromaines et romaines. Gallia - Archéologie de la France antique, 2008, Lattara/Lattes (Hérault) : nouveaux acquis, nouvelles questions sur une ville portuaire protohistorique et romaine, 65, pp.131-149. 10.3406/galia.2008.3337 . hal-01913930

\section{HAL Id: hal-01913930 \\ https://hal.science/hal-01913930}

Submitted on 19 Dec 2019

HAL is a multi-disciplinary open access archive for the deposit and dissemination of scientific research documents, whether they are published or not. The documents may come from teaching and research institutions in France or abroad, or from public or private research centers.
L'archive ouverte pluridisciplinaire HAL, est destinée au dépôt et à la diffusion de documents scientifiques de niveau recherche, publiés ou non, émanant des établissements d'enseignement et de recherche français ou étrangers, des laboratoires publics ou privés.

\section{(이) $\$$}

Distributed under a Creative Commons Attribution - NonCommercial - NoDerivatives| 4.0 


\section{LE PORT DE LATTARA}

\section{Premiers acquis sur les phases préromaines et romaines}

\section{Dominique GARCIA}

Mots-clés. Âge du Fer, époque romaine, port, quai, entrepôt, fortification, stockage, phare, navigation.

Résumé. Du VI ${ }^{e}$ s. av. J.-C. au III s. apr. J.-C. l'agglomération languedocienne de Lattes se développe à l'embouchure du Lez, en bordure de lagune. Outre l'abondant mobilier archéologique importé mis au jour, de nombreux aménagements architecturaux découverts lors des récentes fouilles programmées (quais, pontons, hangar, probablement un phare,...) témoignent du rôle portuaire majeur du site de Lattes. Les vestiges s'étendent sur une surface de près de $2500 \mathrm{~m}^{2}$. L'absence de vestiges antérieurs au II ${ }^{e}$ s. av. J.-C. doit être soulignée. Les structures portuaires sont aménagées progressivement jusqu’à la fin du $I^{e r}$ s. av. J.-C., période pendant laquelle un projet parfaitement planifié est mis en place, associant des débarcadères, des structures de stockage (de vin en vrac ?) et probablement un phare. Entre 75 apr. J.-C. et 225 apr. J.-C., on assiste à un réaménagement des espaces existants, lié à une modification, sinon de la nature du moins du mode de conditionnement de l'objet du commerce. Des activités commerciales semblent se dérouler jusqu'à la fin du III $s$. alors que, pour cette période, les fouilles réalisées intra muros ne livrent que très peu de documents. Peut-on proposer l'hypothèse que le port de Lattara ait survécu à la cité ?

Key-words. Iron Age, Roman period, harbour, quay, warehouse, fortification, storage, lighthouse, shipping.

Abstract. From the $6^{\text {th }}$ century B.C. down to the $3^{\text {rd }}$ century A.D., the Languedocian town of Lattes, is expanding at the mouth of the river Lez, along the lagoon. Beside the numerous imported goods recovered a great number of architectural improvments have been found during the last programmed excavations (quays, embankments, warehouse, probably a lighthouse...) testify to the major role of the site of Lattes as a port. Remains lie on a surface of near 2,500 $\mathrm{m}^{2}$. The lack of remains earlier to the $2^{\text {nd }}$ century B.C. must be pointed. The port structures are progressively equipped until the end of the $1^{\text {st }}$ century B.C. when a project perfectly planned is undertaken, combining unloading areas, storehouses (for wine in bulk ?) and probably a lighthouse. Between 75 and 225 A.D., we are witnessing a restructuring of the existing spaces, linked to a modification, if not to the nature, or at least to the packaging process of trading products. Trading activities seem to go on until the end of the $3^{\text {rd }}$ century A.D. although for this period very few information has been gathered from the excavations conducted intra muros. Can we suggest that the harbour of Lattes lasted after the decline of the city?

Translation: Isabelle FAUDUET

Schlüsselwörter. Eisenzeit, römische Zeit, Hafen, Kai, Lager, Befestigung, Lagerung, Leuchtturm, Schifffahrt.

Zusammenfassung. Vom 6. Jh. v. Chr. bis zum 3. Jh. n. Chr. entfaltet sich Lattes im Languedoc an der Mündung des Flusses Lez, am Rand einer Lagune. Abgesehen von dem hohen Anteil von Importwaren im Fundmaterial zeugen zahlreiche, bei den kürzlich durchgeführten Plangrabungen entdeckte Anlagen (Kais, Pontons, Lager, Leuchtturm?,...) von der bedeutenden Rolle von Lattes als Hafen. Die Befunde verteilen sich über eine Fläche von fast $2500 \mathrm{~m}^{2}$. Das Fehlen von Spuren aus der Zeit vor dem 2. Jh. v. Chr. ist eine Tatsache, die angemerkt werden muss. Die Hafenanlagen werden nach und nach bis in die letzten Jahre des 1. Jahrhunderts v. Chr. ausgebaut, dann entsteht ein perfekt vorgeplantes Hafenprojekt, zu dem Anlegeplätze, Lagerstrukturen (für Wein) und wahrscheinlich ein Leuchtturm gehören. Zwischen 75 und 225 n. Chr. werden die existierenden Anlagen neu gestaltet, diese Umstrukturierung hängt mit der Änderung, wenn nicht mit der Natur der Handelsware, so doch mit deren Konditionierung zusammen. Bis in das ausgehende 3. Jh. scheint der Handel aufrecht erhalten worden zu sein, während die Grabungen intra muros für diese Zeit nur sehr wenig Fundmaterial liefern. Darf man hieraus schließen, dass die Aktivität des Hafens von Lattes die der Stadt überdauert hat?

Übersetzung: Isa ODENHARDT-DONVEZ 


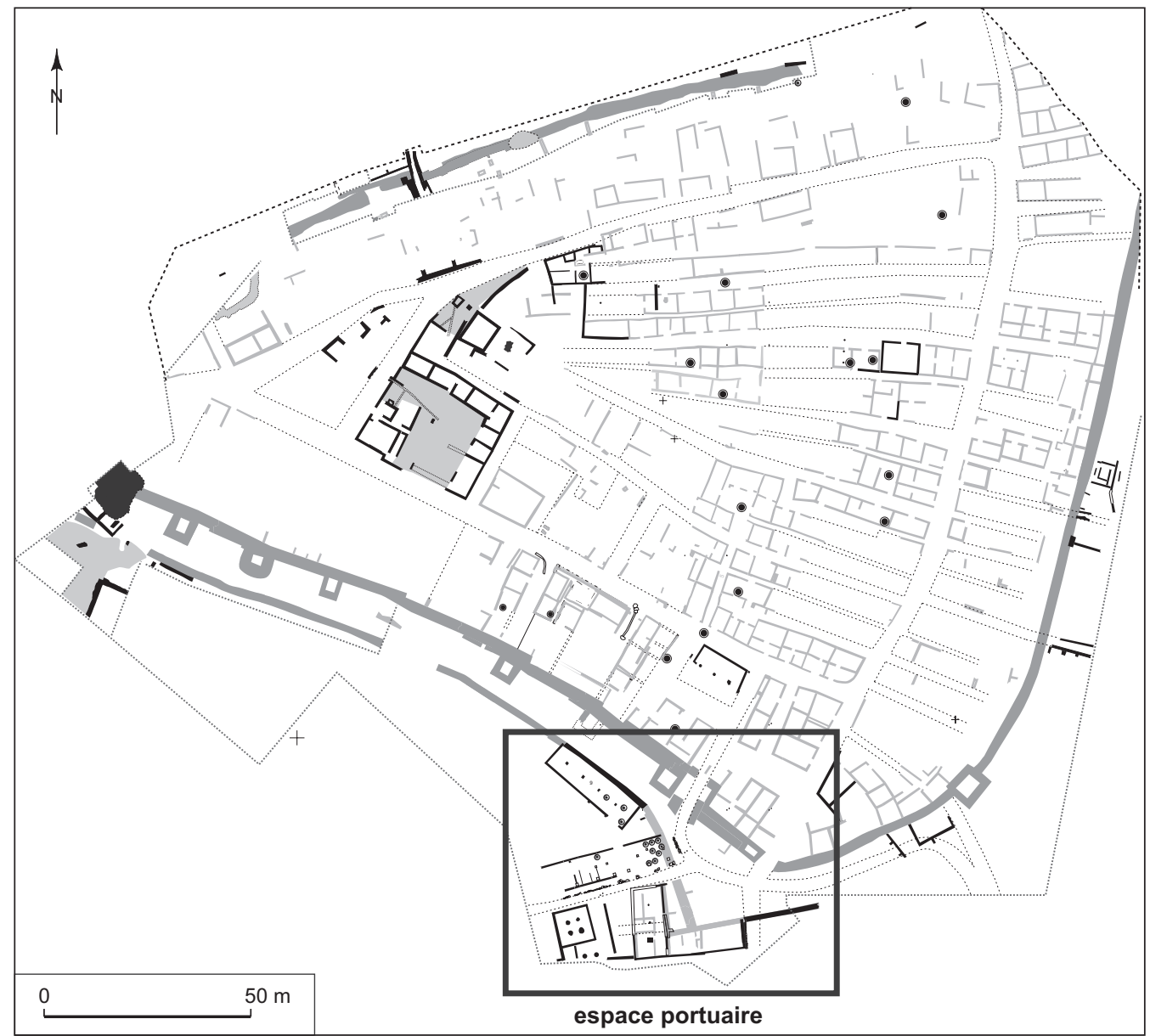

Fig. 93 - Plan général des vestiges mis au jour sur le site de Lattes et localisation de l'espace portuaire (DAO : (C) UFRAL).

\section{LA VILLE « DU FLEUVE ET DU MARAIS »}

Du VI ${ }^{\text {e }}$ s. av. J.-C. au III ${ }^{\text {e }}$ s. apr. J.-C., l'agglomération languedocienne de Lattes se développe à l'embouchure du Lez, en bordure du stagnum Latera (Pline l'Ancien, H. N., IX, 29). Outre l'abondant mobilier archéologique importé mis au jour lors des fouilles, de nombreux aménagements architecturaux découverts lors des fouilles récentes (quais, pontons, hangar, possible phare...) témoignent du rôle portuaire majeur du site (fig. 93 et 94). Nous allons détailler l'évolution architecturale de cet ensemble original après avoir, en quelques lignes, présenté le cadre topographique du port, son lien avec l'habitat et son environnement immédiat.

L'origine gauloise du nom de Lattes (Latera) comme celui d'Arles (Arelate) renvoie au milieu naturel dans lequel sont implantées les deux agglomérations protohistoriques. Pour X. Delamarre, dans les deux cas cités, le toponyme signifie «devant les » ou «près des » (are) «marais » (latis), localisations propres à ces agglomérations protohistoriques (Delamarre, 2001, p. 45 et p. 166). G. Barruol $\mathrm{a}$, quant à lui, proposé de voir dans « are » une référence au fleuve (Barruol, 1988) ; Lattes serait alors la ville du fleuve et du marais. Dans les deux propositions, les caractères naturels se retrouvent dans le nom même de la ville. Peu avant le milieu du $\mathrm{I}^{\mathrm{er}}$ s. apr. J.-C., Pomponius Mela décrit le littoral méditerranéen et associe dans une même phrase les étangs des Volques, le fleuve Lez et le castellum de Latara (Pomponius Mela, De chorographia, II, 80).

La lagune et le fleuve : les deux composantes naturelles organisant l'espace portuaire sont là. En règle générale, la formation du cordon littoral est progressive et, durant la Protohistoire, les passages entre la lagune et la mer devaient être dans bien des cas plus nombreux et plus larges qu'ils ne le sont aujourd'hui. Mais pour Lattes, les Anciens 


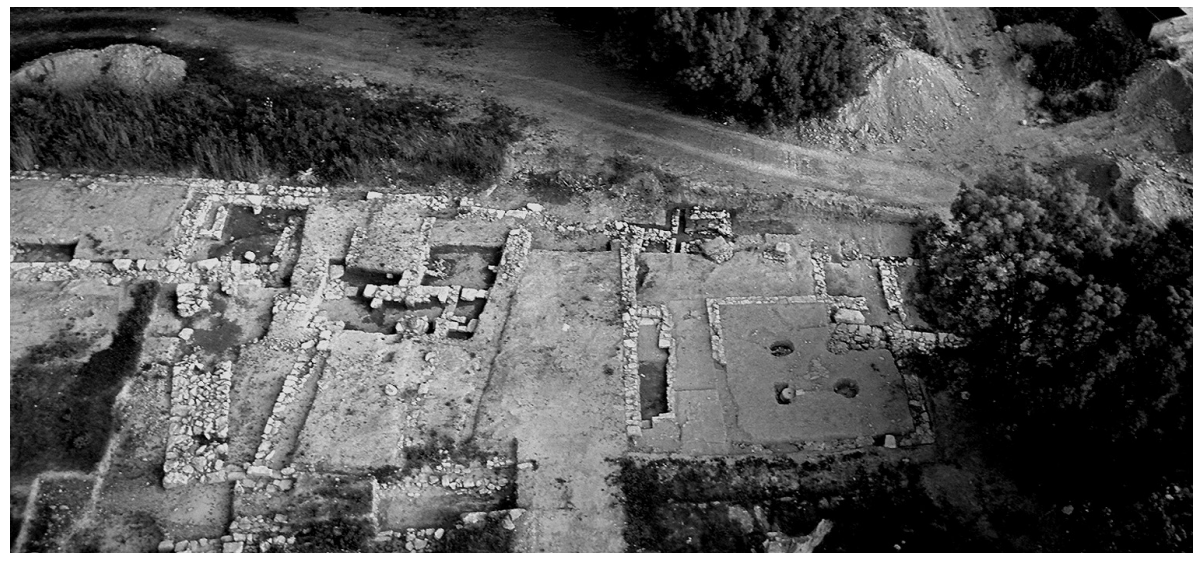

Fig. 94 - Vue générale de la terrasse portuaire de Lattes (prise du nord) (photo : (C UFRAL).

avaient déjà clairement la perception d'un espace séparé de la mer. Pour preuve l'utilisation de l'expression « stagnum Latera» par Pline l'Ancien (H. N., IX, 29) vers 70 apr. J.-C., peut-être même empruntée à des écrits plus anciens (le $D e$ animalibus de Pompeius Trogus?). Les détails fournis par le naturaliste lorsqu'il narre la pêche aux muges sont extrêmement clairs : il fait état de l'étroit goulet reliant la mer à l'étang («angustis faucibus stagni in mare»), seul endroit que l'on pouvait barrer d'un filet ("locumque solum pandendis retibus») et des nombreux espaces profonds (" alto trepidosque») de cette lagune.

Le stagnum Latera est un espace naturel qui peut faire office de façon idéale de bassin portuaire. Il a une profondeur suffisante pour être fréquenté par les bateaux maritimes et, grâce à l'épi formé par le cordon littoral, il constitue un lieu bien protégé de la houle et des tempêtes. En règle générale, l'envasement des bassins est un problème important pour les gestionnaires des ports. A priori, les rejets des eaux du Lez - mêlées d'alluvions - dans l'étang peuvent paraître un inconvénient majeur, mais le courant provoqué par le fleuve, en permanence ou lors des crues saisonnières, a sans doute permis de créer un chenal naturel dans l'axe de son confluent, facilitant ainsi aux bateaux l'accès au rivage. Aujourd'hui, fleuve domestiqué et suraménagé, le Lez - le ledum flumen de Pomponius Mela (De chorographia, II, 5) au I ${ }^{\mathrm{er}}$ s. apr. J.-C. - porte peut-être à travers les différentes acceptions des racines gauloises de son nom (Leto le « reflux », selon Hamlin, 2000, p. 215 ou Leto " gris » ou " limoneux », selon Delamarre, 2001, p. 166) le caractère bouillonnant que lui ont connu naguère les Lattarenses.

Les vallées des fleuves côtiers méditerranéens ont sans aucun doute joué le rôle de voies naturelles de com- munication entre le littoral et l'arrière-pays, et la spécificité du biotope des zones de delta ou de confluence est sans nul doute propice à l'exercice d'activités agropastorales (Lattara, 5 ; Blanchemanche, Chabal, 1995 ; Garcia, 2000). Mais, pour la Protohistoire et le long de cet espace lagunaire, entre la côte rocheuse pyrénéenne et les avancées calcaires de la Provence occidentale, on peut reconnaître dans ces chenaux - ces cours inférieurs des fleuves et leur prolongement à travers les étangs - un attrait supplémentaire pour ces populations dépendantes des contacts et des échanges.

$\mathrm{Vu}$ de la mer, l'accès au stagnum Latera était marqué par le tertre de Maguelone qui devait constituer pour les navigateurs un amer remarquable. Cette île a été fréquentée dans l'Antiquité et dès la Protohistoire (CAG, 34/3, p. 377-380). Dans ce cas également, l'analyse du toponyme, sans constituer une fin en soi, est riche de significations. Pour F. Hamlin, le nom Maguelone est probablement composé des éléments préceltiques mag « hauteur » et lona « marais» (Hamlin, 2000, p. 25). Maguelone serait donc la « hauteur s'élevant des marais » justifiant pleinement sa fonction de repère. Mais n'est-il pas possible de se référer à l'une des formes les plus anciennes (Magolonensium au $\mathrm{V}^{\mathrm{e}}$ s. apr. J.-C. dans la Noticia Galliarum) pour proposer une origine du nom issu des termes gaulois magos (le «marché », Delamarre, 2001, p. 180) et lona (le «marais ») qui associerait Maguelone, l'amer de Lattes, à « l'emporion des étangs "? S'il est pour l'heure injustifié de faire de Maguelone un avant-port de Lattara, nous conserverons comme seule hypothèse que cette île a pu jouer un rôle de repère maritime, marquant la proximité du grau (le passage) qui relie la mer à l'étang. 

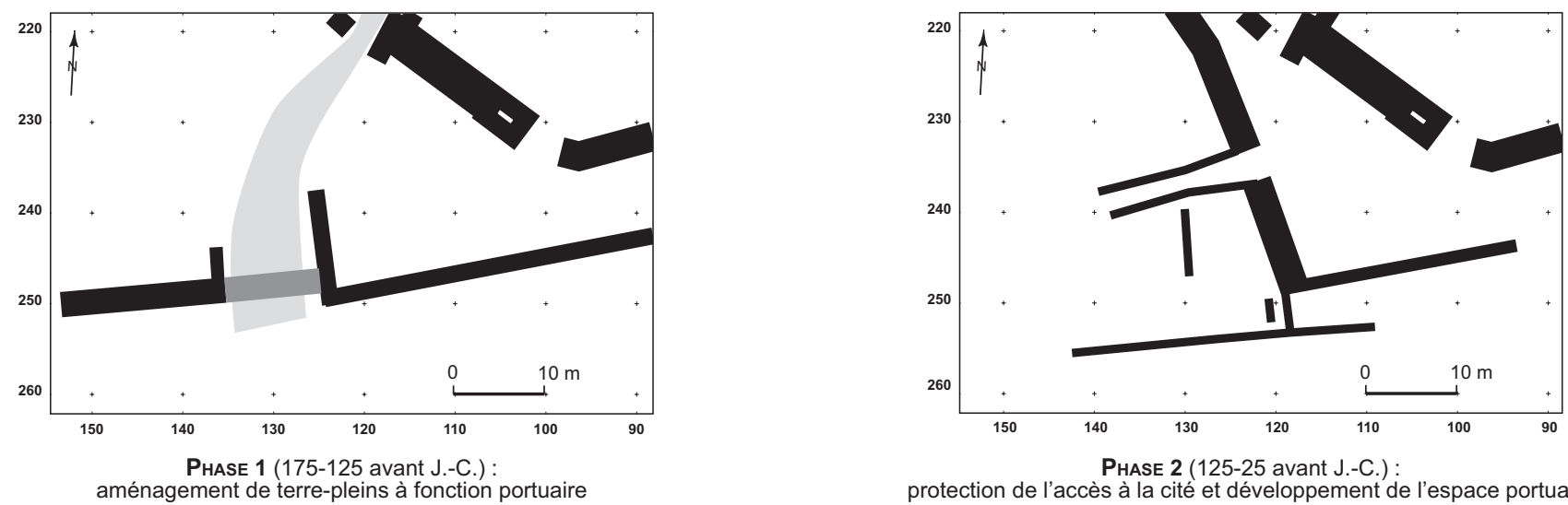

protection de l'accès à la cité et développement de l'espace portuaire
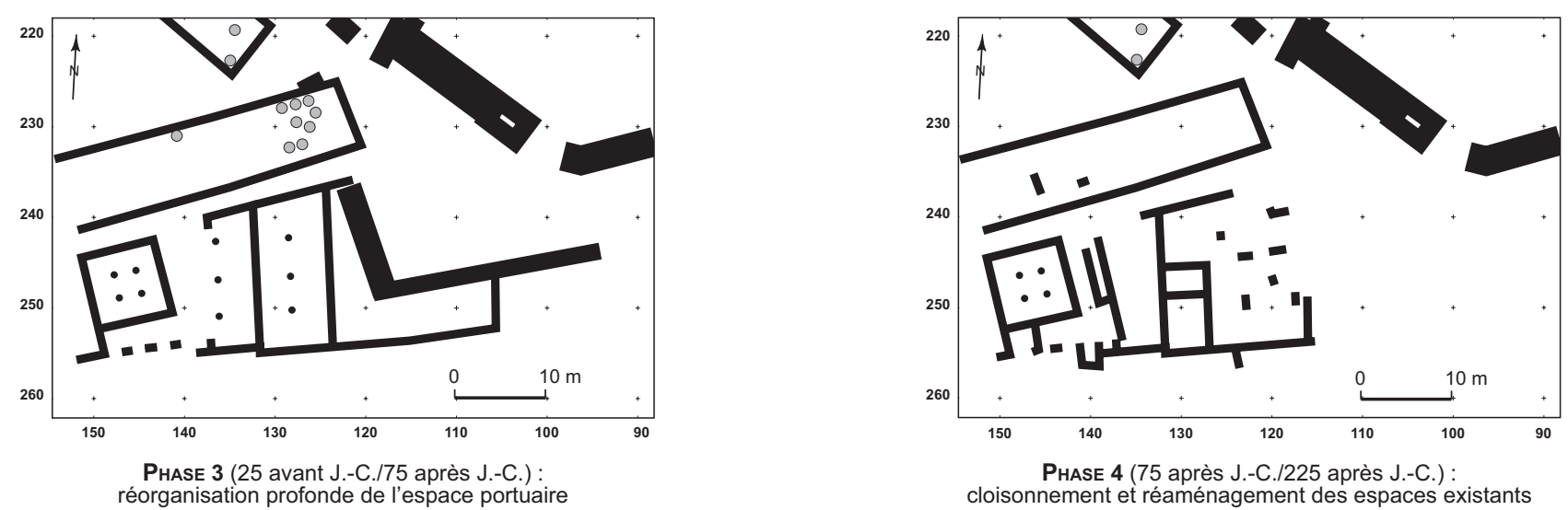

Fig. 95 - Évolution topographique simplifiée de la terrasse portuaire de Lattes du II ${ }^{e}$ s. av. J.-C. au III s. apr. J.-C. (DAO : D. Garcia, Université Aix-Marseille).

\section{LES AMÉNAGEMENTS PORTUAIRES}

L'espace exploré se situe dans la partie méridionale de la parcelle Saint-Sauveur de Lattes, dans la partie sud-est de l'agglomération préromaine, au-delà du rempart et de la rue 124, à l'aboutissement d'un chenal antique du Lez (Jorda, 2000 et 2002). Les vestiges mis au jour s'étendent sur une surface globale de près de $2500 \mathrm{~m}^{2}$ qui a été décapée en plusieurs étapes et a fait l'objet de six campagnes de fouilles d'un mois chacune, réalisées de 1996 à 2001 (Garcia, Vallet dir., 2002).

L'absence de vestiges portuaires antérieurs au $\mathrm{II}^{\mathrm{e}} \mathrm{s}$. av.J.-C. est un premier fait à noter. Les recherches menées dans les années 1960 par Henri Prades et son équipe avaient abouti au même constat. Seuls des niveaux lagunaires profonds (à plus de 3,50 m de profondeur) avaient livré des céramiques étrusques et massaliètes que l'on peut attribuer à la fin $\mathrm{du} \mathrm{VI}^{\mathrm{e}} \mathrm{s}$. av. J.-C. ou à la première moitié $d u \mathrm{~V}^{\mathrm{e}} \mathrm{s}$. av. J.-C. Le port des $\mathrm{V}^{\mathrm{e}} \mathrm{s}$., IV $\mathrm{IV}^{\mathrm{e}} \mathrm{s}$. et $\mathrm{III}^{\mathrm{e}} \mathrm{s}$. av. J.-C. est placé dans un autre secteur de la ville ou bien sa surface est beaucoup plus restreinte et ses vestiges sont à rechercher non loin du parement extérieur de l'enceinte sous les bâtiments que nous avons mis au jour.

Schématiquement, la dynamique générale du développement de la zone portuaire, de la fin de l'âge du Fer à la fin de l'occupation du site de Lattes, peut être illustrée par deux grands points :

- Les aménageurs n'ont eu de cesse de développer des aires de circulation et/ou de stockage en avant du rempart, en limitant au minimum les recharges de remblais ; 


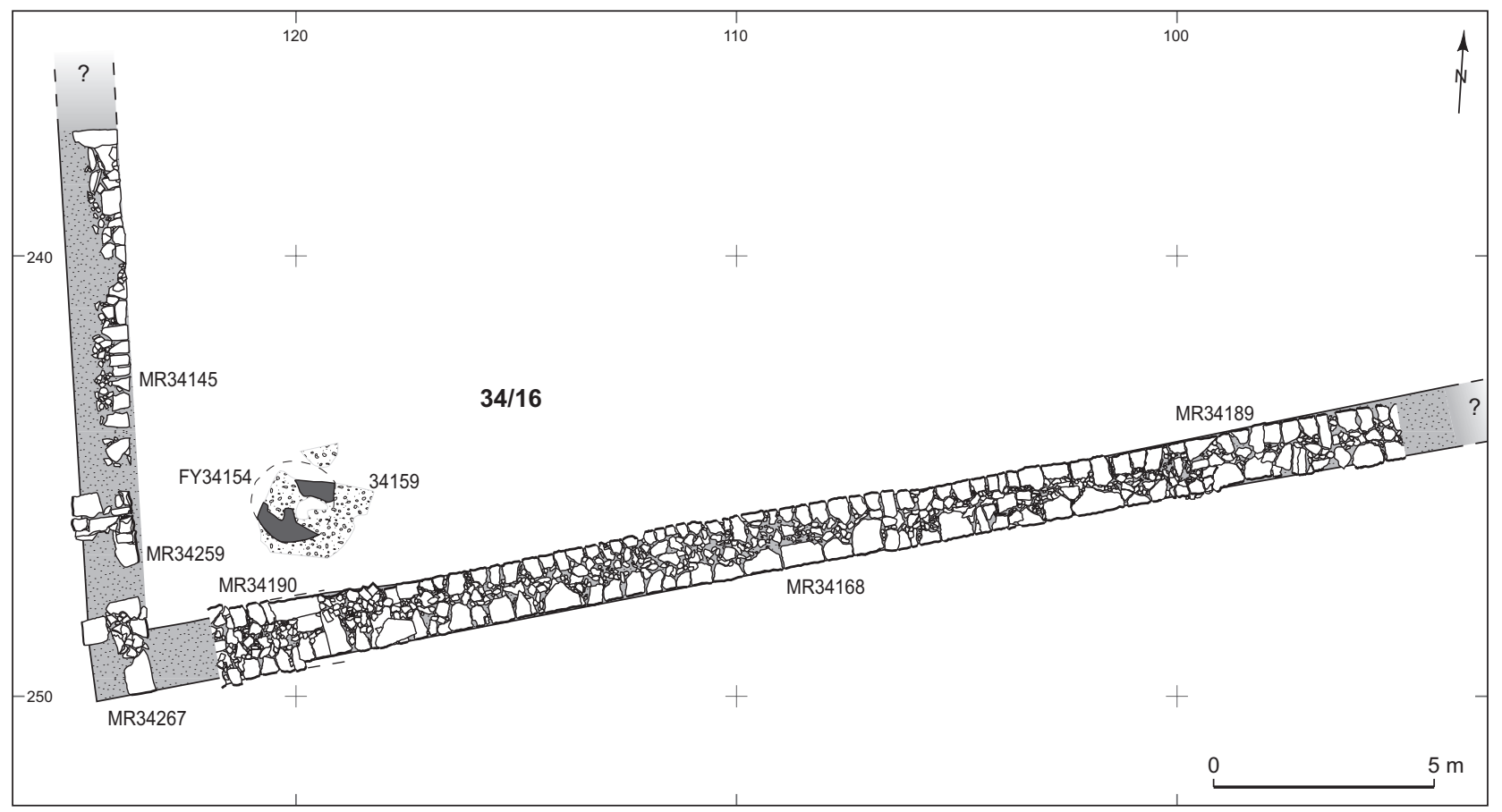

Fig. 96 - Plan général des vestiges de la phase 1 du port de Lattes (DAO : (C) UFRAL).

- Le développement s'est fait par l'aménagement successif de quais en avant du rivage afin de maintenir, voire d'augmenter, la profondeur d'eau nécessaire à l'accostage des navires.

La mise en phase a été effectuée en croisant des données planimétriques et stratigraphiques. Elle a été précisée par l'analyse typochronologique du mobilier mis au jour. Les phases 1 à 4 ont été définies en fonction de modifications architecturales notables tant au point de vue du plan que des fonctions. La phase 5 n'a été mise en lumière que par la présence d'un ensemble clos de mobilier. L'étude fine du mobilier céramique a permis d'illustrer certaines sous-phases, peu sensibles dans l'observation des structures architecturales (en particulier les sous-phases $4 \mathrm{a}$ et 4b). Inversement, l'étude du bâti implique de mettre en avant des sous-phases dont la datation ne peut être précisée au sein d'une fourchette large (par exemple les sous-phases $1 \mathrm{a}, 1 \mathrm{~b}$ et 1c). La phase 5 qui ne se distingue pas par des aménagements architecturaux spécifiques a été traitée en conclusion de la phase 4 (fig. 95).

\section{Chronologie}

Phase 1 : $175-125$ av. J.-C.

Phase 2 : 125-25 av. J.-C.
Phase 3 : 25 av. J.-C./75 apr. J.-C.

Phase 3a : 25 av. J.-C./- 1 av. J.-C.

Phase 3b: 1 av. J.-C./75 apr. J.-C.

Phase 4 : 75 apr. J.-C./225 apr. J.-C.

Phase 4a : 75 apr. J.-C./175 apr. J.-C.

Phase 4b : 175 apr. J.-C./225 apr. J.-C.

Phase 5 : 225 apr. J.-C./300 apr. J.-C.

\section{PHASE 1 (175-125 AV.J.-C.) : AMÉNAGEMENT DE TERRE-PLEINS À FONGTION PORTUAIRE}

L'intervalle chronologique 175-125 av. J.-C. correspond à la date de fonctionnement la plus basse pour les aménagements mis au jour, en bordure de la lagune antique, au sud des fortifications méridionales de la ville préromaine. Ces structures peuvent être plus anciennes, ou faire suite à des constructions plus anciennes que nos fouilles n'ont pas révélées (fig. 96). L'analyse de cette phase correspond à l'étude d'une aire de fouille d'environ $1020 \mathrm{~m}^{2}$ (60 m d'est en ouest et $17 \mathrm{~m} \mathrm{du}$ nord au sud) pour laquelle des observations ont été faites en respectant les états architecturaux postérieurs. Elle ne résulte donc pas à proprement parler d'une fouille en aire ouverte. En dehors des structures les plus conséquentes que nous avons repérées, il est fort probable que des aménagements 


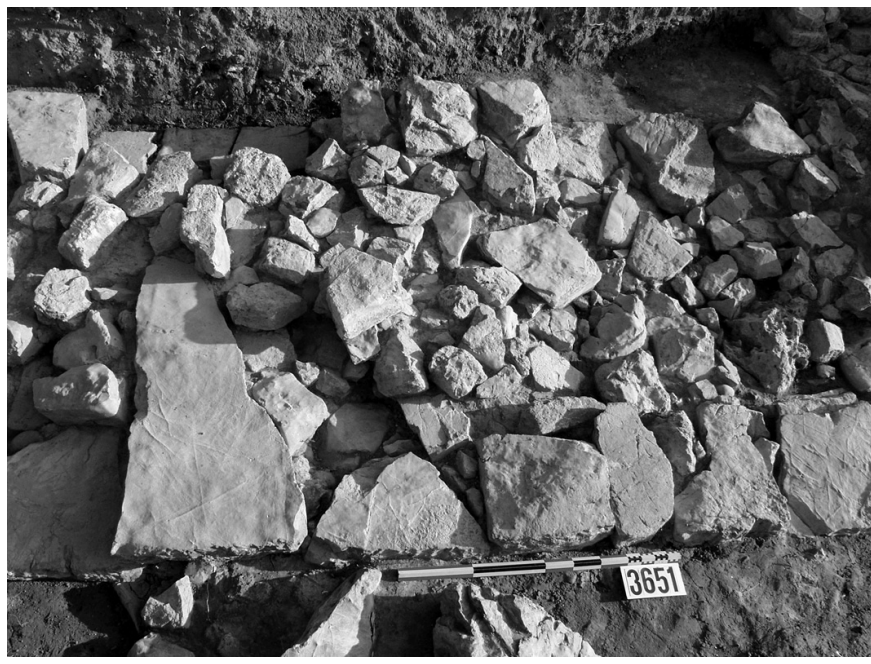

Fig. 97 - Détail de l'appareillage du mur-quai MR34211 du port de Lattes (photo : (C) UFRAL).

complémentaires échappent à notre étude. L'interprétation $\mathrm{du}$ fonctionnement de cette phase primitive repose sur un nombre élevé d'hypothèses, mais la documentation de base n'est pas à négliger pour autant. En effet, en contexte équivalent, les structures suburbaines contemporaines sont rarissimes et les documents lattois permettent, pour la première fois en Méditerranée nord-occidentale, d'appréhender un espace portuaire protohistorique. Dans l'état actuel de nos recherches, plusieurs pièces essentielles à l'analyse de ce type de dossier peuvent être mises en avant. Premièrement, il existait des constructions extra muros en bordure de la lagune au moins dès la fin du second âge du Fer. Ces structures ne se présentent pas sous la forme de bâtiments mais comme des terre-pleins d'au moins $1000 \mathrm{~m}^{2}$, aménagés devant la porte méridionale de la ville et retenus par deux murs d'environ 1,30 m d'épaisseur. Ce type de construction, en gagnant sur un espace sublagunaire, permettait de se rapprocher des zones de plus forte profondeur et réservait en amont des espaces voués à la circulation, au chargement et au déchargement des marchandises et à d'autres activités.

Si ces constructions possédaient une forte élévation, on peut envisager qu'elles revêtissent une fonction défensive en doublant le rempart et en protégeant la porte. Si leur hauteur ne dépassait guère l'état conservé aujourd'hui, c'est plutôt une fonction portuaire que l'on peut proposer : nous aurions là un quai. La largeur des murs, notamment celle du mur MR34168, peut laisser supposer une surface de circulation sur son assise supérieure. Ainsi, à titre d'hypothèse, cette construction pouvait revêtir trois usages distincts et complémentaires : maintien de remblais constituant une aire de circulation et de services devant la porte méridionale de la cité, circulation de personnes et de véhicules sur sa surface, mur de quai sur lequel viendraient s'appuyer des appontements en direction du large.

Il convient cependant de rester prudent car la terrasse retenue par ces constructions n'a été observée que sur moins de $20 \mathrm{~m}^{2}$ (secteur 16), dans l'angle constitué par les deux murs. On a relevé un sol de galets associé à un foyer et un remblai en partie fouillé contenant un mobilier daté des années 175-125 av. J.-C. Un tel aménagement (sol de galets et foyer) ne doit pas forcément être associé à un espace couvert. Les importantes dimensions du foyer et sa position (non loin du parement interne du mur de soutènement) ne plaident pas en faveur d'un usage domestique. On peut penser que le sol de galets correspond à une aire de circulation aménagée en surface de la terrasse, devant la porte. Le foyer pourrait être lié à une activité de fabrication ou de réparation mais aussi marquer, en tant que fanal, la présence du quai et de la porte méridionale de l'agglomération.

Lors d'une deuxième sous-phase (1b), de peu postérieure à la sous-phase 1a, on note la construction d'un mur à double parement (MR34211) mis au jour dans les secteurs 21 et 23. Il s'agit d'une construction bien conservée, orientée est-ouest, dégagée sur une quinzaine de mètres de longueur et accusant une largeur moyenne de 2,20 m. Nous ne connaissons pas son extrémité occidentale. Vers l'est, ce mur a été observé dans le secteur 21 alors qu'il est absent dans le secteur 24. Tout oriente vers l'hypothèse d'un retour vers le nord. Même s'il est sensiblement plus large que le mur MR34168, sa mise en œuvre et la nature pétrographique des blocs incitent à rapprocher ces structures. Les deux murs ont la même direction, mais ne sont pas alignés : MR34211 (fig. 97) est construit 1,20 m plus au nord que MR34168. Le mur MR34211 pourrait être le pendant occidental du mur MR34168. Entre ces deux aménagements, un passage en pente douce (une cale ?), d'une dizaine de mètres de large, aurait pu être aménagé vers la lagune. Cette hypothèse peut être amendée par le niveau de graviers accusant un pendage vers le sud, observé dans le secteur 24. La troisième sous-phase correspond au bouchage du passage situé entre les deux murs.

\section{PHASE 2 (125-25 AV. J.-C.) : PROTECTION DE L'ACGÈS À LA GITÉ ET DÉVELOPPEMENT DE L'ESPACE PORTUAIRE}

Cet état de la terrasse portuaire correspond à deux sous-phases architecturales. La première (2a) est relative à la construction d'un mur réutilisant en partie le mur 


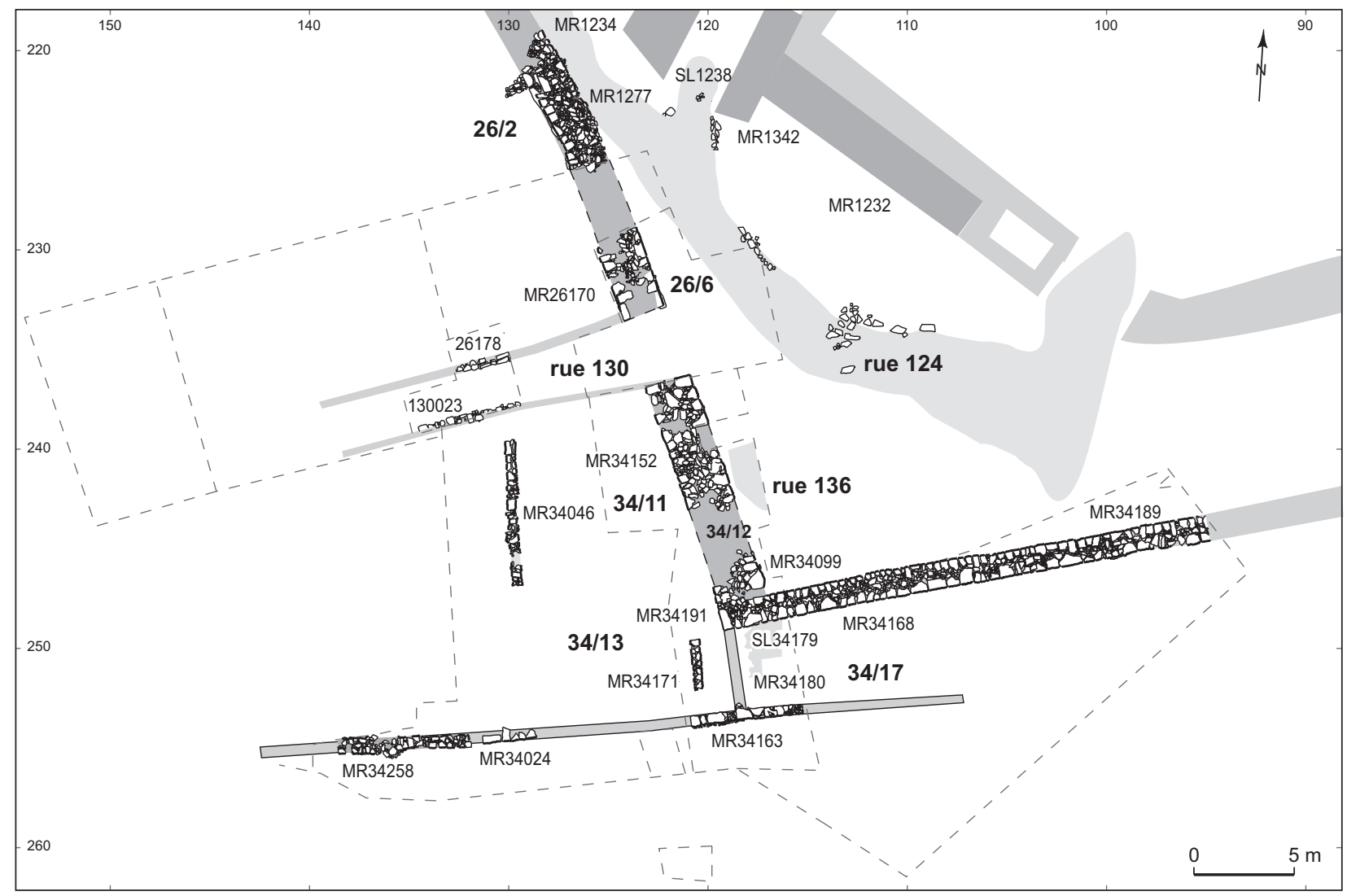

Fig. 98 - Plan général des vestiges de la phase 2 du port de Lattes (DAO : (C) UFRAL).

MR34168 et qui vient doubler le rempart tout en limitant un terre-plein devant la porte 23/8. Le tronçon septentrional de ce mur d'enceinte [26177] s'achève vers le sud par un piédroit. Il a une largeur de 2,20 m et peut être rapproché de l'état ancien de l'avant-mur ( $c f$. Lattara, 9, p. 51). Le niveau d'arasement de ce mur a été observé par J.-C. Bessac pour qui il ne fait aucun doute que la destruction du mur n'a pas été suivie d'un remblaiement rapide de la structure. Bien au contraire, il semblerait, d'après les traces de poli visibles sur l'épiderme des blocs, que l'on ait circulé activement sur cet arasement. Il est tentant de dater cette phase de circulation entre les années 75 et 25 av. J.-C. L'espace entre les deux tronçons de mur est de 3,40 m ; aucun système de fermeture n'a été observé. Cette porte constitue la limite orientale de la rue 130. Dans l'angle formé par les murs, un lambeau de rue, ou plus probablement une aire de circulation aménagée en bordure de la rue 124, a été fouillé. Cet ensemble forme un doublage du rempart qui renforce ainsi la protection de la ville et limite un terre-plein devant la porte 23/8 (fig. 98).
Dans un deuxième temps (2b), à l'ouest et au sud de la rue 130 , on note un agrandissement sensible de la terrasse portuaire primitive. Après arasement des principales structures antérieures, le secteur est remblayé de façon homogène. Ce remblai est retenu au sud par le mur MR34027, d'une largeur moyenne de $0,55 \mathrm{~m}$, situé environ $4 \mathrm{~m}$ au sud des anciens murs de terrasse. Notre vision des aménagements présents sur ce terre-plein au sud et nord de la rue 130 est particulièrement lacunaire : la quasi-totalité des constructions et des sols sont soit arasés, soit cachés par des structures postérieures qui n'ont pas été démontées lors de la fouille. Cette phase d'occupation est donc marquée par une volonté de protéger l'entrée méridionale de la ville et de réserver un espace de service d'environ $300 \mathrm{~m}^{2}$ entre les deux murs de protection. En avant de ces structures, le terre-plein gagné sur la lagune est agrandi ; il occupe une surface de $800 \mathrm{~m}^{2}$ au minimum. Plusieurs constructions, probablement des bâtiments de type hangars, sont alors créées de part et d'autre d'une rue. L'accès à l'eau ne paraît plus pouvoir se faire directement, du moins dans la partie que nous avons explorée. 


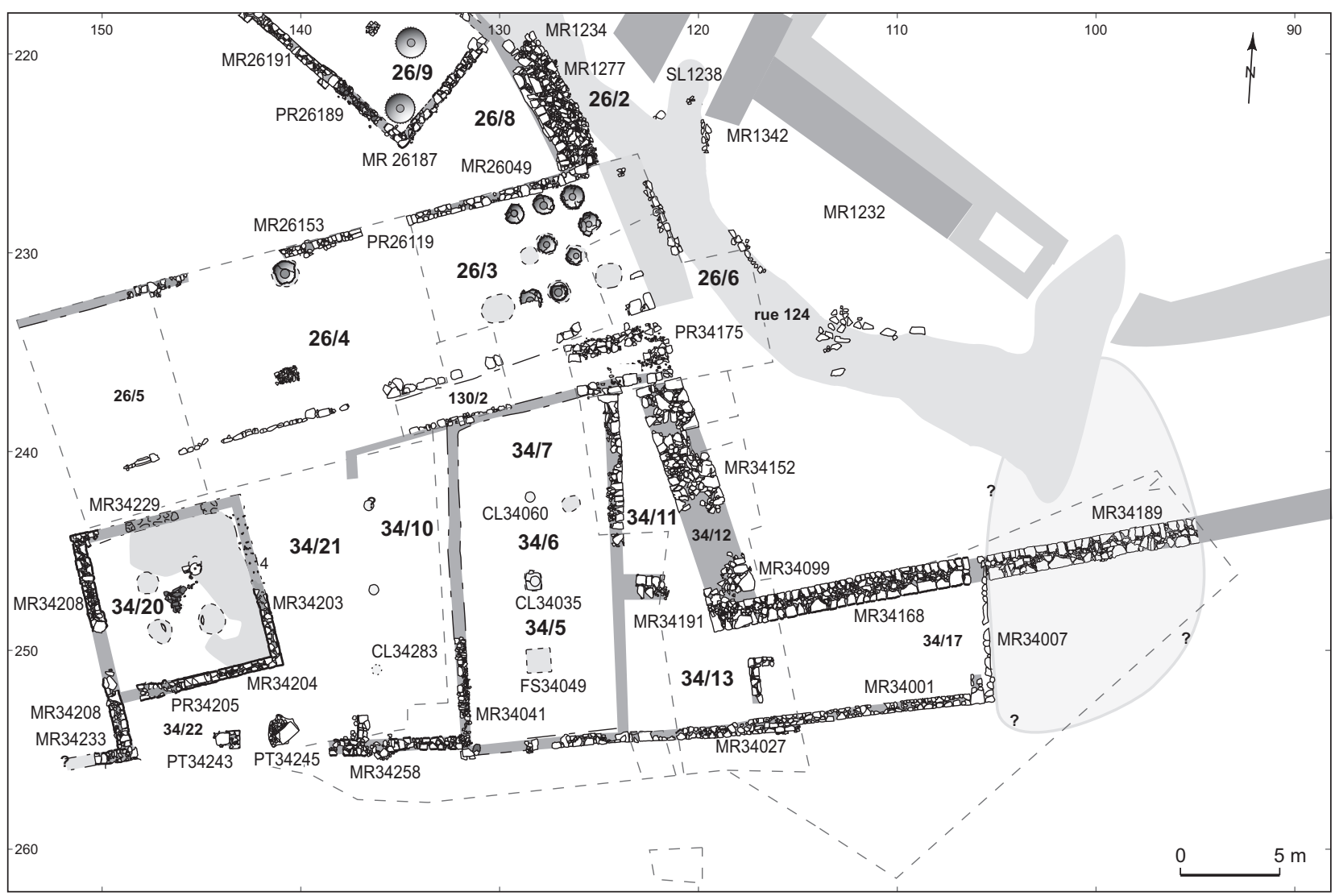

Fig. 99 - Plan général des vestiges de la phase 3 du port de Lattes (DAO : (C) UFRAL).

\section{PHASE 3 (25 AV. J.-C./75 APR. J.-C.) : RÉORGANISATION PROFONDE DE L'ESPACE PORTUAIRE}

Cette phase correspond à l'ensemble architectural le mieux conservé, dont la lecture reflète un projet parfaitement planifié. Les deux sous-phases adoptées font référence, d'une part à la mise en place (3a) des différents bâtiments dans le courant du dernier quart du I ${ }^{\mathrm{er}} \mathrm{s}$. av. J.-C., plus probablement dans la dernière décennie de cette fourchette chronologique et, d'autre part, à l'utilisation primaire de ces bâtiments durant les trois premiers quarts du I ${ }^{\mathrm{er}}$ s. av. J.-C. (3b) (fig. 99).

Au nord de la rue 130 (zone 26), sont aménagés deux bâtiments de stockage en dolia: le bâtiment 26/3-6 placé en bordure septentrionale de la rue 136 et le bâtiment 26/9 appuyé contre l'avant-mur MR1277. Au sud de la rue 130, la terrasse est alors retenue par le mur MR34001. Sur cette terrasse, on retrouve d'est en ouest : une pièce oblongue
(34/17) qui existait déjà pendant la phase 2, un espace trapézoïdal (34/13), un grand bâtiment rectangulaire hypostyle (34/5-7), un portique (34/10), un passage vers la lagune $(34 / 21)$ et enfin un édifice à plan carré $(34 / 20)$ prolongé d'un porche $(34 / 22)$.

Le mur de soutènement principal (MR34001) orienté nord-est/sud-ouest est large de $53 \mathrm{~cm}$. Ce mur est fondé sous le niveau actuel de la nappe phréatique et nous avons pu l'observer de façon continue sur une longueur d'environ $16 \mathrm{~m}$ et en reprise ou en négatif sur une longueur supplémentaire de $19 \mathrm{~m}$. Son élévation est réalisée en pierres assisées (cailloux, moellons et blocs calcaires) liées par un mortier blanc ; elle est conservée sur une hauteur de cinq assises. Un retour de ce mur, orienté nord-ouest/sud-est, a été partiellement dégagé vers l'est ; il pourrait marquer la limite d'un espace de circulation de la porte préromaine vers la lagune (à l'est de ce retour, le mur MR34189 est alors partiellement arasé).

Le bâtiment à dolia (26/3-6) a connu une occupation assez longue. Cet espace a été dégagé sur toute sa largeur 
(au nord de la rue 130), soit entre 7,50 m et $9 \mathrm{~m}$, et sur une longueur de $30 \mathrm{~m}$. Son extrémité sud-ouest n'a pas été atteinte avec certitude ; la présence d'un fossé agricole - qui marque l'actuelle limite de la parcelle - a perturbé les niveaux fouillés dans ce secteur. Il s'agit donc d'un bâtiment de plan rectangulaire d'au moins $255 \mathrm{~m}^{2}$. C'est une pièce oblongue, limitée sur l'un des longs côtés par un mur percé en son centre d'une porte charretière de 2,35 m de large, et largement ouverte sur ses deux autres côtés connus.

Le petit côté documenté, qui donne sur la rue 124, se présente sous la forme d'un alignement de blocs parementés d'un seul côté, qui limite le podium du bâtiment. En façade sur la rue 130, vers le sud-est, la bordure du podium est marquée par un alignement discontinu de rangées de blocs également parementées d'un seul côté. Une de ces structures (SB26152), la plus occidentale, remploie un fragment de base de colonne engagée de gros module renvoyant au type corinthien (le type «Vernègues » de R. Ginouvès, datable des années 40-30 av. J.-C.). Un sondage réalisé sous cette base a montré que ces aménagements reposent sur une dense couche de remblai datable du dernier quart du $\mathrm{I}^{\mathrm{er}}$ s. av. J.-C.

La toiture de ce bâtiment - un hangar ouvert sur deux côtés au moins - devait reposer sur le long mur de fond et sur une série d'au moins cinq structures verticales placées en retrait de la façade dans des fosses qui avaient un entraxe de 5,30 $\mathrm{m}$; une distance équivalente sépare l'axe des supports et l'axe du mur de fond sur lequel devaient reposer les poutres. Trois emplacements de ces supports ont été découverts dans la partie fouillée. La toiture en tegulae et imbrices (comme le prouvent les nombreux fragments mis au jour) devait déborder jusqu'à l'aplomb de la façade du bâtiment. Du côté est, il est probable que le passage entre les piliers et le mur était protégé par un appentis auquel pourrait appartenir un calage de poteau placé dans l'alignement des blocs.

À l'intérieur du bâtiment, plus précisément entre les emplacements des supports verticaux et le mur de fond, une série de huit fonds de dolia enterrés dans le sol a été découverte (fig. 100). Un autre fond de dolium a été repéré dans le secteur 4 . Une fosse, creusée au centre du secteur 3, pourrait correspondre à un ancien dolium. Les faces internes des parois et des fonds de dolia portent des traces de poissage ; dans l'Antiquité, ce type de traitement était exclusivement réservé aux récipients devant contenir $\mathrm{du}$ vin. Le diamètre interne du fond des dolia varie de $35 \mathrm{~cm}$ à $42 \mathrm{~cm}$; l'altitude de ces fonds varie de plus d'une

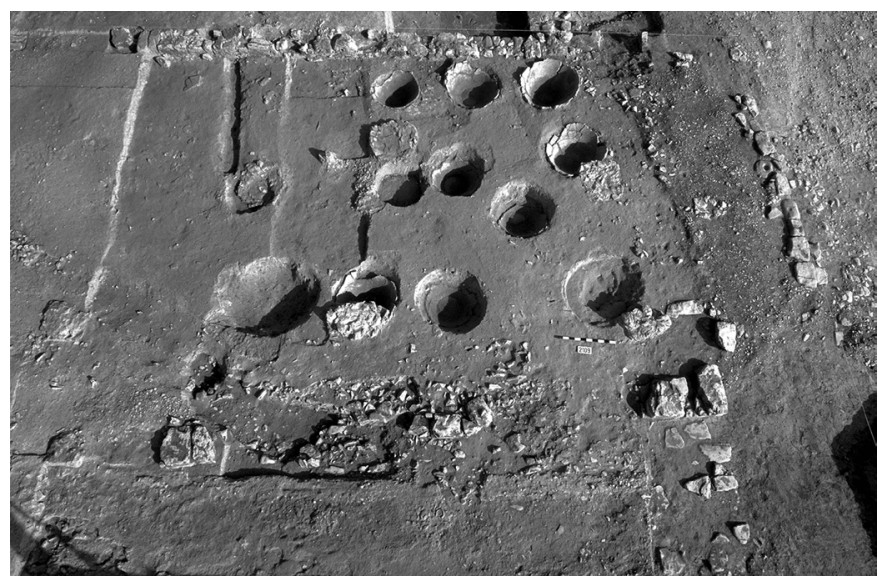

Fig. 100 - Le secteur 3 (cellier) de la zone 26 du port de Lattes en fin de fouille (photo : (C) UFRAL).

dizaine de centimètres, ce qui laisse supposer que ces vaisseaux, de différents types, étaient calés dans des fosses à différents niveaux afin que leur ouverture se place à une même hauteur. Au vu des exemplaires conservés, on peut estimer la capacité maximale du hangar à environ 35/40 dolia. La typologie de ces récipients renvoie à des contenants d'environ 1000 litres, soit une capacité de stockage de 9000 litres dans le cas d'un remplissage maximum des dolia conservés et une capacité de stockage maximale de 400 hectolitres si l'on estime que le hangar ait pu contenir 40 dolia.

Le hangar 26/9 est un vaste bâtiment (25 m de long sur $6 \mathrm{~m}$ de large) parallèle au rempart. Le grand mur de façade (MR26191) était percé par au moins deux portes (dont une large de 3,30 m) et renforcé par des contreforts dont deux ont été conservés. Aucun niveau de sol fonctionnant avec le bâtiment n'a été retrouvé ; en revanche, quelques structures particulières, aménagées dans un remblai de limon jaune, ont pu être renseignées : quatre bases de poteau disposées à $5 \mathrm{~m}$ les unes des autres, un dépôt de fondation composé de deux lampes à huile, de deux vases à paroi fine, d'une aiguille en os, d'une monnaie et de pignons de pin, trois dolia non poissés. Ce hangar était probablement destiné au stockage de denrées diverses dans des dolia mais aussi dans d'autres récipients dont on n'a pas retrouvé la trace. Sa localisation ne permettait pas un déchargement direct à partir des bateaux et c'est plutôt un rôle de dock ou de magasin que nous lui attribuerons.

Entre le hangar (26/9) que nous venons de décrire et le grand bâtiment à dolia (26/3-6), il existe un espace (secteur 8) triangulaire non couvert, de 11,70 m de long sur 7,50 m de large. 
Au sud de la rue 130, on note l'aménagement d'une série d'ouvrages que nous décrirons d'ouest en est : un édifice à plan carré (34/20) prolongé par un porche (34/22), un passage vers la lagune $(34 / 21)$, un portique $(34 / 10)$, un grand bâtiment rectangulaire hypostyle (34/5-7), un espace trapézoïdal (34/13), et enfin une pièce oblongue $(34 / 17)$ qui existait déjà pendant la phase 2.

Vers l'ouest, le premier bâtiment fouillé (34/20) est un ouvrage carré de $80 \mathrm{~m}^{2}$, orienté nord-ouest/sud-est. Le mur sud est percé d'une porte. On peut envisager - mais sans argument tangible - l'existence d'une seconde porte dans l'axe de celle décrite ci-dessus, dans le mur nord et ouvrant sur la rue 130. Devant ce bâtiment, on a noté la présence de deux importantes bases de piliers et on suppose l'existence d'une troisième à l'ouest, dont on n'a pas trouvé la trace. La présence de ces piliers laisse à penser soit que le mur de terrasse était implanté plus au sud, soit qu'il existait plutôt en cet endroit une pente douce vers la lagune. Ces imposantes bases de piliers suggèrent l'existence d'un porche, ce qui donnait au bâtiment une allure générale rectangulaire (12 m x 9 m). Au sud-ouest, devant le mur MR34233 d'orientation est-ouest, on a observé une surface de circulation composée de pierres, de galets et de tessons. À l'intérieur du bâtiment, on a mis en évidence plusieurs aménagements : quatre bases de piliers ou de colonnes, un sol en galets et un foyer central. Les quatre bases (dont trois sont des éléments architecturaux récupérés) se répartissent de manière symétrique à égale distance les unes des autres : deux dans le prolongement du piédroit ouest de la porte PR34205 et les deux autres dans le prolongement du piédroit est de cette porte. Les bases devaient appartenir à un bâtiment antérieur non localisé actuellement et ont été réemployées à la fin du $\mathrm{I}^{\mathrm{er}}$ s. av. J.-C. Ces éléments architecturaux appartiennent à un ou plusieurs bâtiments publics monumentaux. Il s'agit de bases italo-corinthiennes de type «Vernègues » que l'on date communément des années 40-30 av. J.-C. et que l'on retrouve par exemple dans la phase primitive de la «maison au Dauphin » de Vaison-la-Romaine. Le bâtiment auquel elles appartenaient aurait donc eu une durée de vie assez courte, inférieure à une génération. Au centre du carré d'environ 2,50 m de côté que forment ces bases, on a noté la présence d'une sole de pierres brutes en grès qui a subi une forte rubéfaction. Un sol de galets a été observé le long des murs. Ce sol, affaissé au niveau du seuil, semble avoir laissé libre la partie centrale de la pièce, permettant la circulation autour du foyer, ce qui permet de supposer que le bâtiment n'était peut-être pas couvert au centre, l'ensemble des quatre colonnes ou piliers supportant un lanterneau.
La fonction de cette construction n'est pas simple à interpréter (fig. 101). Il s'agit d'un bâtiment à plan centré, largement ouvert sur la lagune dont la surface utile est largement entamée par un foyer ceinturé d'un sol de galets. On notera l'absence de structure de stockage, de banquette, d'aménagements domestiques ou artisanaux. L'activité principale de cette pièce semble liée à ce foyer, placé au centre de la pièce et dans l'axe de l'ouverture. Les quatre bases de colonnes permettaient très probablement de maintenir un lanterneau. La fonction cultuelle - en l'absence d'aménagements ou de matériel spécifiques semble devoir être écartée. L'organisation spatiale de l'espace est peu propice à un lieu de réunion. Aussi, suivant ainsi la proposition faite par plusieurs collègues spécialistes d'archéologie maritime, nous suggérons d'interpréter cet édifice comme un phare directionnel. Dans l'axe de la porte, le feu placé sur le sol sensiblement plus élevé que le niveau de la lagune pouvait être perçu de loin et guider les navigateurs dans leur approche de l'espace portuaire lattois, en leur indiquant l'axe de pénétration le plus propice vers le quai. En cela, il est bien plus qu'un simple «fanal » (comme celui proposé pour la phase 1), jouant le rôle d'amer et destiné à prévenir les bateaux d'un danger ou de la proximité d'infrastructures portuaires. Ce phare directionnel pouvait indiquer au marin l'axe du chenal à suivre. Ce type de feu directionnel a récemment été signalé pour la période romaine dans la lagune de Venise et de nombreux exemples modernes ou subcontemporains sont attestés, notamment dans la région des étangs. En effet, le faible tirant d'eau dans la lagune obligeait les navigateurs à maintenir un cap précis. Le port étant placé à l'embouchure d'un bras du Lez, ou à proximité de celle-ci, c'est très probablement le fleuve lui-même qui créait, dans l'axe de son cours, le chenal le plus favorable à la navigation. En l'absence de comparaison architecturale, c'est sans doute une meilleure connaissance de la topographie antique de la bordure de la lagune qui permettra d'appuyer ou d'infirmer cette hypothèse.

Entre le bâtiment que nous venons de décrire et le mur de terrasse se trouve un espace ouvert de plan trapézoïdal (secteur 34/21), très probablement une zone de passage entre la rue 130 et la lagune, comme en témoigne la présence d'un épais remblai accusant un pendage vers le sud. Ce passage donnait également accès au bâtiment 34/20 et au portique $(34 / 10)$ placé plus à l'est. Du point de vue de la topographie générale de la zone, cet espace permettait de relier des ouvrages ayant des directions légèrement différentes. En effet, le bâtiment 34/20 est perpendiculaire à la 


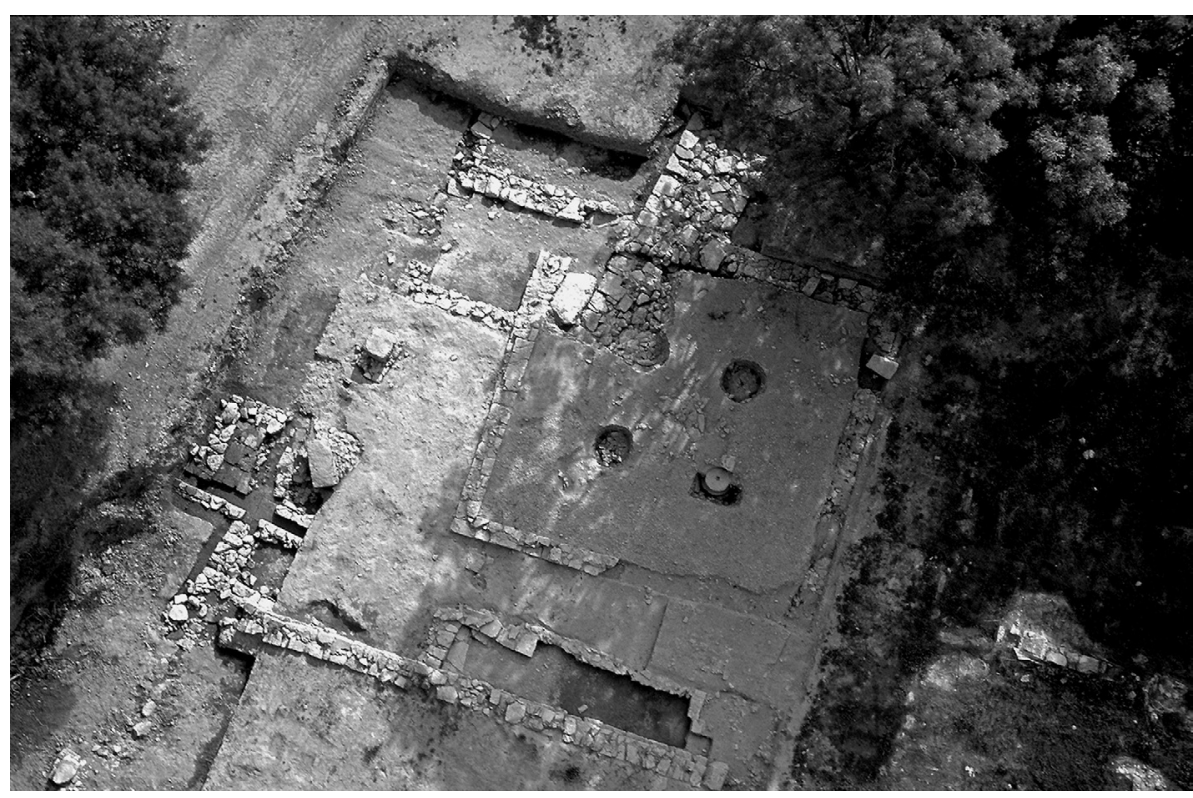

Fig. 101 - Vue aérienne du probable phare directionnel du port de Lattes (photo : (C) UFRAL).

rue 130 alors que les autres constructions sont, quant à elles, perpendiculaires au mur de terrasse.

Àl'est du passage existait vraisemblablement un portique $(34 / 10)$ largement ouvert vers l'ouest : deux tambours de colonne en pierre du Bois-des-Lens ont été réutilisés comme bases ; ils pourraient marquer la façade d'un édifice qui comportait à l'origine trois supports verticaux en façade. La présence d'un angle chaîné au sud-est est un indice qui pourrait suggérer que le bâtiment 34/10 est un ajout au plan initial et que l'espace de circulation 34/20 a pu être plus large dans un état primitif. Aucun niveau de sol de l'utilisation initiale de ce bâtiment n'a été conservé. Une fonction de hangar peut être proposée, sans aucune certitude.

L'édifice suivant est un grand bâtiment rectangulaire de $136 \mathrm{~m}^{2}$ (16,50 m x 8,25 m, soit exactement un rapport de 1 sur 2), orienté nord-sud, dont le mur méridional vient s'appuyer en bordure de la terrasse contre le parement interne du mur MR34001. L'ampleur de l'épierrement dont il a fait l'objet ne permet pas de localiser l'accès au bâtiment ; la porte pouvait se trouver soit au nord, en façade sur la rue 130, soit à l'est comme pourrait le suggérer la présence de deux blocs de calcaire qui pourraient correspondre à un piédroit. Ce bâtiment, probablement couvert de tuiles, possédait très certainement une toiture à double pente soutenue par trois colonnes ou poteaux qui reposaient sur des supports placés dans l'axe du bâtiment selon un entraxe de 4,20 m. Aucun niveau de sol de l'utilisation initiale n'a été conservé. Seul un fond de fosse localisé dans la partie nord-est pourrait être interprété comme un indice d'utilisation, en l'occurrence une activité de stockage qui rapprocherait ce bâtiment de celui fouillé au nord de la rue 130. En tout état de cause, la localisation du bâtiment, sa technique de construction et sa surface plaident en faveur d'une fonction commerciale et particulièrement de stockage.

À l'est semble avoir été établi un espace non couvert (34/11) épousant la forme d'un trapèze très irrégulier. On a mis au jour le seuil d'une porte ( $1 \mathrm{~m}$ sur $0,55 \mathrm{~m}$ ) qui s'ouvre sur la rue 130 . Le dernier espace que l'on peut sommairement situer est le secteur 34/17. L'absence d'aménagement sur un sol limono-argileux incite à l'interpréter comme un lieu non couvert, annexe au secteur 34/13. Côté oriental, tout indique que le mur MR34189 a été largement épierré, au moins sur une douzaine de mètres, puis remblayé, probablement pour pratiquer une zone de passage vers la lagune.

$\mathrm{Au}$ terme de la description des aménagements de cette phase, plusieurs constats peuvent être établis. Cet état reflète un projet architectural cohérent, même s'il reprend et réutilise des aménagements et des constructions plus anciennes. La terrasse portuaire, au sens large du terme, a été sensiblement agrandie : au vu des espaces fouillés, on peut estimer sa surface à un minimum de $2400 \mathrm{~m}^{2}$ au lieu des $1200 \mathrm{~m}^{2}$ de la phase antérieure (dont $400 \mathrm{~m}^{2}$ d'espace de service en avant de la porte ancienne). Le rempart de la 
ville n'est plus doublé. Certains indices laissent même penser qu'il est en partie démantelé. Les orientations des bâtiments sont dictées par deux directions qui convergent vers l'ouest : celle de la rue 130 et celle du mur de terrasse MR34001. On notera qu'au sud de la rue 130 toutes les constructions sont organisées par rapport au mur de terrasse MR34001, à l'exception du « phare directionnel » qui est perpendiculaire à la rue. Ces constructions sont bâties selon un module de base identique de $9 \mathrm{~m}$ de côté que l'on peut retrouver dans le plan du secteur 34/20 dont le plan carré fait 9 m de côté extra muros. Ainsi on peut attribuer au bâtiment 26/3-5 des proportions de 1 module sur 4 , au bâtiment $34 / 20$ celles de 1 module de côté (un tiers de module supplémentaire pour le porche), au bâtiment $34 / 10$ celle de $1 / 2$ module de large et 2 de long, au bâtiment 34/5-7 celles de 1 de large et 2 de long, à l'espace 34/11-13 celles de 1 de large sur 2 de long... Les techniques de constructions rapprochent également les différents ouvrages. Lorsqu'elles sont conservées, les élévations sont en moellons grossièrement taillés liés au mortier de chaux de couleur blanche. Les fondations sont généralement liées à la terre sauf celle du mur septentrional de la pièce 34/20 faite de mortier et de pierres coulés dans une tranchée. Les sols sont en terre, généralement des remblais à base de limon argileux. Les remplois sont fréquents, notamment les bases de piliers qui réutilisent des éléments d'architecture de bâtiments publics du début de la seconde moitié du $\mathrm{I}^{\mathrm{er}}$ s. av. J.-C. Ces témoins attestent une phase d'architecture monumentale tardo-hellénistique dont la durée d'utilisation aura été inférieure à une génération.

Tous ces éléments conduisent à interpréter cet état comme un reflet de la romanisation du site de Lattes : dans les techniques de construction, les formes architecturales mais également les fonctions. L'usage essentiel des constructions mises au jour semble être lié au stockage de denrées. C'est l'usage que l'on peut attribuer certainement au bâtiment 26/3-5, probablement en partie au bâtiment 26/8 et hypothétiquement à l'édifice 34/5-7. La fonction des espaces 34/10 et 34/11-13-17 ne peut être précisée ; la structure 34/20-22 pourrait être, quant à elle, un phare directionnel. L'accès à ces espaces se faisait principalement par la rue 130, sur laquelle sont visibles des traces de circulation d'attelages. L'accès au rivage était possible soit par l'espace 34/21, soit à l'extrémité orientale de la zone. Comme nous l'avons laissé entendre, nous interprétons les bâtiments de stockage comme étant destinés, pour tout ou partie, au stockage du vin en vrac. En effet, tant la forme générale des constructions (en particulier le bâtiment 26/3-5) que leur équipement (la présence de dolia dans les bâtiments 26/3-5 et 26/8) nous conduit à les rapprocher de structures clairement identifiées et interprétées comme des chais de transbordement (Hesnard, 1997). Passons en revue les éléments appuyant une telle interprétation. Sur plusieurs dolia du bâtiment 26/3-5 sont conservées des traces de poix, ce qui interdit d'y voir d'une part des récipients destinés à contenir des denrées solides, des céréales en particulier, mais également d'autre part, des vases de stockage d'huile d'olive dont l'acidité attaquerait l'enduit poissé. L'usage des dolia pour le stockage du vin est largement reconnu par l'archéologie et les textes. On retrouve le plus souvent le dolium isolé ou en quelques exemplaires pour un usage domestique ou dans le coin d'une boutique. La présence d'un grand nombre de vaisseaux dans un même bâtiment est attestée dans les chais aménagés à proximité des structures de production. De nombreuses villae de Narbonnaise, par exemple, ont livré de tels aménagements destinés le plus souvent à la fermentation du moût et au «vieillissement » du vin jusqu'au printemps suivant la récolte. Ensuite, le vin est transvasé dans des récipients aptes à la commercialisation et au transport par voie terrestre, fluviale ou maritime : outres, tonneaux ou, le plus souvent, amphores. La localisation des ouvrages lattois dans la zone portuaire, bien loin des vignobles et, dans l'état actuel de la documentation, d'éventuelles structures de pressurage, ne plaide pas en faveur d'un ou plusieurs chais liés à une production locale de vin. La forme même des bâtiments 26/3-5 et 26/8, largement ouverts sur l'un de leurs grands côtés, n'est pas à notre connaissance attestée pour des lieux de fermentation ou de vieillissement du vin. Plus que des chais, nos bâtiments sont à interpréter comme des docks. La mise en évidence, tant par la palynologie que par la carpologie et, depuis peu, par des traces agraires, d'un vignoble important dès la fin de l'âge du Fer, aux portes septentrionales de la ville, pourrait inciter à lier cette production viticole lattoise aux docks viticoles de la zone portuaire : le vin serait transporté dans ces hangars avant d'être transvasé dans des amphores ou placé dans des dolia chargés sur des bateaux. Une telle hypothèse ne peut être totalement écartée, mais il semble plus logique d'insérer nos découvertes tant par leur chronologie que par le plan des vestiges dans un schéma explicatif plus large, documenté par de nombreuses découvertes terrestres et maritimes. En effet, on doit en particulier à A. Hesnard d'avoir mis en relation les découvertes d'entrepôts portuaires équipés de dolia poissés avec des épaves armées de dolia à poste fixe et d'avoir interprété ces vestiges comme le témoignage d'un transport et d'un stockage de vin en vrac (Hesnard et al., 1988 ; Hesnard, 1997). 
« Les entrepôts portuaires à dolia sont connus depuis les grandes fouilles d'Ostie et les fouilles de F. Benoit à Marseille, dans l'immédiat après-guerre. Longtemps mal interprétés - entrepôts à blé -, ils sont depuis une vingtaine d'années mis en relation avec des navires contenant des dolia à poste fixe, dont plusieurs épaves ont été découvertes et fouillées. La fouille de nouveaux entrepôts à Marseille (place Jules-Verne) permet de confirmer les hypothèses émises à propos des épaves et d'affiner la problématique concernant ce type de transport ». D’après les données actuelles, « de l'époque augustéenne jusque vers le milieu du $\mathrm{II}^{\mathrm{e}}$ s. apr. J.-C., une flotte de navires spécialisés transporte du vin en vrac, versé dans les dolia des navires dans le port d'embarquement, puis vidé dans le port de destination " (Hesnard, 1997, p. 130). Des entrepôts de ce type sont connus en quatre exemplaires à Marseille (Hesnard, 1994, p. 212 et n. 37), à Ostie, à Luni (cité dans Hesnard, 1997), à Aquilée (travaux en cours de M.-B. Carre), à Lyon en bordure de la Saône (Audin, 1968), etc. Plus près de Lattes, à Agathé (Agde), nous rappellerons la découverte ancienne d'une série de dolia interprétée comme les vestiges d'un entrepôt (Clavel, 1970, repris par Bedon, 2001, p. 47). En Méditerranée, une douzaine d'épaves de bateaux à dolia sont signalées, dont celle du Grand Ribaud (Hesnard et al., 1988), de Ladispoli (Gianfrotta, Hesnard, 1987), ou de la Giraglia (Marlier, à paraître). On notera que dans ces épaves ont également été retrouvées des amphores de Pompéi et de Tarraconaise. Plus près de Lattes, peut-être peut-on rapprocher la découverte «d'une batterie de plusieurs dolia en place dans le port même de Marseillan » avec la présence d'une épave de ce type (Ambert et al., 1993, p. 132). Le vin serait en provenance de différents vignobles d'Italie (Adriatique, Étrurie, Campanie...) et serait destiné au marché gaulois où il pouvait être redistribué en amphores. Les nombreuses amphores à fond plat associées à des couvercles mis au jour dans le hangar 26/3-5 ont pu avoir cet usage (Sanchez, Adroher, 2002).

Ainsi les découvertes lattoises prennent dans ce contexte une place particulière. Elles sont le témoignage occidental de ce type d'entrepôt, donc de commerce, et attestent que ce trafic a également touché des ports secondaires par rapport à Marseille, Ostie ou Lyon. Cette interprétation des vestiges met également l'accent sur un point essentiel : celui de la relative autonomie de la dynamique d'évolution des structures portuaires par rapport à la cité de Lattes. En effet, les aménagements qui touchent le port dans le dernier quart du I ${ }^{\text {er }}$ s. av. J.-C. (modification des quais, construction ou réfection profonde des bâtiments de stockage...) sont liés à des phénomènes qui dépassent largement le cadre local. Les navires à dolia ont un tirant d'eau estimé à 1,60 m et nécessitent des aménagements portuaires standards, qu'ils soient à Lattes ou à Marseille. La phase architecturale que nous avons étudiée est certainement le reflet du dynamisme du port alors même que la ville tend à se désagréger, mais plus encore elle est la démonstration de la participation de ce port à un trafic extrêmement large. Il est peu probable que le vin qui arrive à Lattes fût destiné à la seule consommation locale et il n'est pas impossible que le site ait maintenu, comme durant l'âge du Fer, un rôle de redistribution de vin méditerranéen en Languedoc oriental, dans l'est de la cité de Nîmes en particulier.

\section{PHASE 4 (75 APR. J.-C./225 APR. J.-C.) : CLOISONNEMENT ET RÉAMÉNAGEMENT DES ESPACES EXISTANTS}

La quatrième phase d'occupation de la terrasse portuaire correspond à un réaménagement profond de la zone, en particulier par une série de cloisonnements internes des espaces existants à l'exception du bâtiment quadrangulaire (secteur 34/20) (fig. 102). Deux sous-phases ont été mises en évidence grâce au mobilier: phases $4 \mathrm{a}$ (75 apr. J.-C./175 apr. J.-C.) et 4b (175 apr. J.-C./225 apr. J.-C). Le bâtiment 26/3-5 a livré les traces de nombreuses modifications : si la surface de l'édifice reste la même, son organisation générale, son élévation et peut-être sa fonction sont alors modifiées. En effet, les dolia sont tous arasés à un niveau identique et comblés dans le dernier quart du I ${ }^{\mathrm{er}}$ s. apr. J.-C., la toiture est mise à bas, les structures porteuses (colonnes ?) sont récupérées et les deux fosses sont rapidement remplies de déchets, notamment d'amphores de type Gauloise 1. De nouveaux supports vont être ensuite mis en place : en témoignent deux séries de fosses quadrangulaires comblées de petits blocs noyés dans du mortier. Ces aménagements devaient supporter des poteaux de bois, peut-être de section quadrangulaire comme le suggère l'empreinte laissée par l'un d'entre eux dans le mortier. Une première rangée est régulièrement alignée en retrait d'environ $2 \mathrm{~m}$ par rapport à la façade. Leur entraxe est d'environ 4,50-5 m, excepté entre deux supports où il est seulement de 3,50 m. Cette anomalie pourrait être liée au fait que l'on voulait éviter d'avoir un poteau dans l'axe de la porte. Entre cette rangée de supports et le mur de fond se trouve une autre rangée de structures de diverses natures, disposées moins régulièrement. Si la première rangée pouvait supporter les piliers de façade d'une toiture probablement en appentis, les 


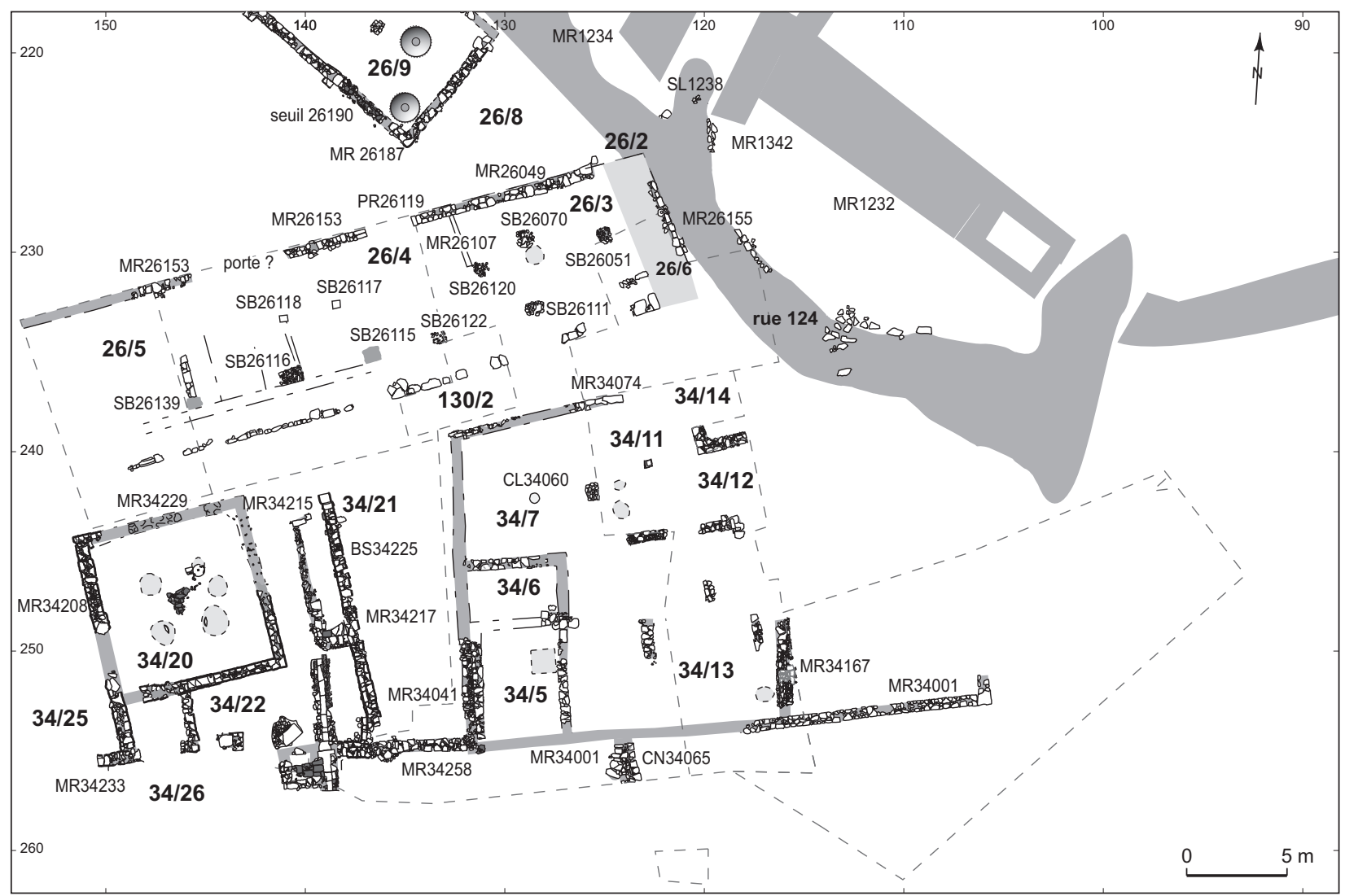

Fig. 102 - Plan général des vestiges de la phase 4 du port de Lattes (DAO : (C) UFRAL).

supports intermédiaires marquent sans doute une volonté postérieure de renforcer la charpente.

La deuxième modification profonde par rapport à l'état antérieur réside dans l'accès et la circulation dans le bâtiment. En effet, la porte précédente est toujours en usage et une seconde porte est sans doute percée, plus à l'ouest, dans l'axe du secteur 4. Pendant cette phase, l'aménagement d'un mur parallèle à la rue observé sur moins d'une dizaine de mètres, environ $1,50 \mathrm{~m}$ en retrait de la façade, témoigne d'un accès privilégié au secteur 4 par les portes percées dans le mur nord. D'autres cloisonnements perpendiculaires à ce mur sont aménagés. Le mur parallèle à la façade vient s'appuyer contre des supports ; on construit ensuite des parois plus étroites et perpendiculaires à ce mur. Deux d'entre elles limitent une pièce de 2,50 m sur 2,90 m contre les parois de laquelle s'appuient deux banquettes assez larges (1,20 m et 1,60 m) et hautes d'une quinzaine de centimètres, réservant un espace central profond de 1,10 m. Les niveaux en place de cette pièce étaient recouverts par un remblai très compact qui scellait un niveau d'effondre- ment d'enduits muraux écrasés à plat. Cette phase peut-être datée des années 50-110 apr. J.-C.

La surface du bâtiment 26/3-5 est mal conservée et il est difficile de tirer des conclusions tranchées sur l'organisation interne et l'usage de ce bâtiment à partir de la fin $d u I^{e r}$ s. apr. J.-C. Cependant, on peut formuler quelques remarques à partir de nos données (notamment celle du secteur 4) en les intégrant à celles recueillies sur d'autres sites similaires. En premier lieu, on constatera que l'abandon du bâtiment à dolia dans son usage primitif est concomitant à d'autres structures identiques, à Marseille notamment. C'est également durant la fin du I ${ }^{\mathrm{er}}$ s. apr. J.-C. que les bateaux à dolia ne semblent plus naviguer. Le transport du vin en vrac dans des bateaux contenant des dolia à poste fixe et ensuite stocké dans des chais équipés de dolia disparaît. À partir des années 75-100 apr. J.-C., le vin devait être systématiquement conditionné dans des récipients (amphores, outres, tonneaux...), puis entreposé dans des locaux adaptés dont les aménagements du bâtiment 26/3-5 reflètent sans doute le plan. La présence de cloisons 
facilite sans doute le maintien de rangées de récipients. Les banquettes aménagées dans le secteur 4 isolaient les contenants de l'humidité tout en facilitant la manutention. Leur largeur, entre 1,20 m et 1,70 m, peut difficilement correspondre à celle d'amphores disposées à plat, mais elle convient pour appuyer les unes contre les autres des amphores placées debout. Cependant, le plan de cette pièce n'est pas sans rappeler celui des chais à barriques, attestés à des périodes plus récentes. De par la nature même du matériau, le transport et le stockage du vin en tonneaux dans l'Antiquité restent mal connus mais des travaux récents précisent les modalités d'usage et la diffusion de ces pratiques (Barrata, 1997 ; Desbat, 1997 ; Tchernia, 1997). Les tonneaux, dont l'attribution de l'invention aux Celtes est discutée, ont été largement utilisés par les Romains, notamment pour exporter du vin dans les camps militaires de la région rhénane dès la période augustéenne (Desbat, 1997, p. 118). En Gaule méridionale, les textes témoignent de l'utilisation des tonneaux dès le $\mathrm{I}^{\mathrm{er}} \mathrm{s}$. av. J.-C. Lors du siège de Marseille par César, les Grecs «mettent le feu à des barriques remplies de résine et de poix qu'ils font rouler du rempart sur la galerie » (B. G., II, 11, 2). À Lattes même, et de façon exceptionnelle pour un site méridional, plusieurs éléments de tonneaux ont été reconnus. Un exemplaire a été réutilisé comme cuvelage du puits 2 du sondage 26 du groupe archéologique Painlevé, creusé au I ${ }^{\mathrm{er}}$ s. av. J.-C. ? (Py, 1988, p. 101-102, fig. 21 et 22). Il avait un diamètre d'environ $0,90 \mathrm{~m}$ et une hauteur minimum de $1 \mathrm{~m}$. Une bonde a été retrouvée par $\mathrm{H}$. Prades lors des fouilles du port dans son sondage VI (Arnal et al., 1974, p. 64). C'est également la fonction que l'on peut attribuer au bouchon en buis que nous avons mis au jour dans le sondage 2 de la zone 26. Les dimensions prises sur des tonneaux antiques conservés correspondent à celles des banquettes que nous avons dégagées. En effet, la majorité des tonneaux conservés a une hauteur comprise entre $90 \mathrm{~cm}$ et $210 \mathrm{~cm}$ et un diamètre de $70 \mathrm{~cm}$ à 110 cm (Baratta, 1997, p. 109 ; Desbat, 1997, p. 115). Sans exclure d'autres solutions, nous pouvons donc proposer que tout ou partie du hangar pût servir au stockage de récipients remplis de vin, dont des tonneaux. Pour A. Desbat : "les bateaux à dolia ont pu avoir leur pendant dans des bateaux à tonneaux [...], des vins courants d'Espagne ou d'Italie ont pu gagner la Gaule et la Germanie autrement qu'en amphores »(Desbat, 1997, p. 117). Face aux nombreuses contraintes techniques liées au transport du vin en vrac dans des dolia, l'exportation du vin en tonneau a pu se généraliser à partir de la fin du $\mathrm{I}^{\mathrm{er}} \mathrm{s}$. av. J.-C. et conduire ainsi à des transformations dans l'organisation des docks.
Aucun aménagement spécifique ni niveau de sol daté des phases 4 ou 5 n'a été reconnu dans le hangar 26/8. Nous avons cependant dégagé une couche de remblai à base de matériaux rapportés incluant de nombreux fragments de tuiles, enduits, blocs, cailloutis... (niveau daté des années 100-125 apr. J.-C.) qui recouvrait le sol initial. Seul le remplissage secondaire des dolia témoigne d'une utilisation de ce hangar durant cette période et jusqu'à la fin du $\mathrm{III}^{\mathrm{e}} \mathrm{s}$.

La phase 4 correspond à un réaménagement profond de la zone 34. Des remblais sont apportés afin de niveler la terrasse, soutenue au sud par le mur MR34027. Sur cette terrasse sont bâtis des murs avec lesquels aucun niveau de sol n'a pu être mis en relation. Au sud, les grands hangars initialement dévolus au stockage sont compartimentés, tout comme le hangar de la zone 26. À l'ouest du mur MR34041, le mur de terrasse est prolongé par des constructions qui viennent barrer le plan incliné vers le rivage. Des aménagements observés au sud du mur de terrasse (caniveau, niveau de circulation...), en bordure de la limite de fouille, indiquent que durant cette période l'aménagement de la zone progresse vers le sud.

On note ensuite la transformation complète des constructions aménagées sur la terrasse portuaire. Le grand bâtiment à fonction commerciale est détruit et, dans la partie méridionale, le terrain est décaissé d'une cinquantaine de centimètres. Cet événement est révélé de manière très claire par la spoliation du support qui se trouvait dans la fosse FS34049 et la mise au jour d'un niveau de circulation nettement caractérisé dans le secteur 5. Ce décaissement a enfin provoqué un déchaussement des parties méridionales de certains murs qui sont alors doublés vers l'est.

Du nord vers le sud, l'espace nouvellement aménagé pourrait se présenter comme suit : on trouve en bordure de la rue 130 une grande pièce (secteur 7) de plan carré (7 m x $7 \mathrm{~m}$ ) qui pourrait posséder une entrée vers l'ouest. À partir de cette première pièce, on accédait par un passage large de $2,50 \mathrm{~m}$ à un secteur de forme allongée (9 m x 2,50 m), orienté nord-sud, couloir ou passage à l'air libre, probablement en plan incliné vers le sud. Ce corridor distribue deux pièces. La première (secteur 6 ), la plus septentrionale, a une largeur de 2,50 m pour une longueur de 4,50 m. L'absence de niveau de sol conservé ne permet pas d'apprécier sa fonction. La seconde pièce (secteur 5 ) est un espace rectangulaire $(3,70 \mathrm{~m}$ x $5 \mathrm{~m})$, orienté nord-sud.

La partie orientale de la zone a été fortement bouleversée par des aménagements postérieurs et des travaux 
agricoles, mais elle semble posséder un plan assez proche de la partie occidentale. Au nord, un espace probablement non couvert (secteurs 11-12) jouxte au sud une construction dont on a uniquement repéré l'angle, qui donnait sur le secteur 7 à l'ouest et peut-être sur un espace de circulation orienté nord-sud, en bordure orientale de la zone fouillée. De cet espace, on pouvait accéder à deux pièces : l'une rectangulaire et l'autre de plan presque carré (secteur 13).

La fonction de cet ensemble de pièces n'est pas complètement précisée. Cette compartimentation d'un espace initialement dévolu au stockage n'est pas sans rappeler la genèse du grand hangar de la zone 26. C'est d'ailleurs très certainement à la même période (c'est-à-dire dans le dernier quart du I ${ }^{\mathrm{er}}$ s. apr. J.-C.) que ces réaménagements se sont produits. La nature des vestiges, il est vrai très fortement perturbés, ne plaide pas en faveur de constructions à fonction d'habitation.

Comme nous l'avons signalé, le bâtiment quadrangulaire (secteur 20) aménagé à la période augustéenne paraît encore en élévation, peut-être avec une fonction différente. Des constructions partiellement reconnues viennent alors s'accoler au sud de ce bâtiment.

Nous notons donc, durant la phase 4, la transformation importante des constructions aménagées antérieurement sur la terrasse portuaire. Au sud de la rue 130 , si le probable phare est conservé dans un état très proche de son plan d'origine, il n'en va pas de même des autres bâtiments qui sont largement compartimentés. Des constructions possédant des sols de niveaux différents sont aménagées au sein des architectures antérieures. Bassin et caniveaux sont peut-être le témoignage d'activités artisanales. Pour le moins, ils attestent des pratiques qui n'étaient pas documentées lors de la phase précédente, quant les bâtiments étaient, semble-t-il, exclusivement voués au stockage.

$\mathrm{Au}$ nord de la rue 130, l'organisation interne et l'usage du bâtiment 26/3-5 sont modifiés. Nous avons suggéré d'y voir un bâtiment voué au stockage de vin en containers, peut-être des tonneaux disposés sur les banquettes des cellules.

Pour le bâtiment 28/9, la nouveauté principale pour cette phase résulte de l'analyse du mobilier contenu dans les dolia qui montre que l'activité de stockage était encore en cours à la fin du $\mathrm{III}^{\mathrm{e}} \mathrm{s}$. Pour cette période, les fouilles réalisées intra muros ne livrent que très peu de documents. Peut-on proposer que la fonction portuaire du site de Lattes ait perduré à la cité ?

\section{LES FONCTIONS PORTUAIRES}

Le port de Lattes devait être polyvalent mais certaines fonctions - et parfois les aménagements qui leur sont associés - méritent d'être précisées. En premier lieu, il faut éliminer certains usages reconnus dans le monde grec, mais difficiles à percevoir pour un site indigène dont, en particulier, la fonction militaire. Une flotte militaire gauloise spécialisée est peu probable. L'activité de piraterie, attestée chez les Ligures par les auteurs anciens, est davantage à mettre au compte du commerce que de la guerre. Le port était sans doute un lieu plus difficile à défendre qu'un espace militaire en tant que tel. C'est sans doute pour cette raison que, pendant une grande partie de la Protohistoire, les biens sont visiblement stockés intra muros. Les aménagements de la phase 2 (avant-mur) témoignent de cette volonté de renforcer la défense de la porte d'accès au site donnant sur le port.

Le port nous paraît avant toute chose un lieu d'interface commerciale. C'est l'espace où les biens « changent de mains ». La liste des produits qui ont été débarqués à Lattes serait bien trop longue à établir. Mais il faut en premier lieu citer le vin qui, dès les origines de la cité, représente un volume d'importation considérable, comme en témoigne la mise au jour de milliers d'amphores étrusques, grecques ou romaines mais également puniques et ibériques, le plus souvent accompagnées des éléments du service à boisson (Lattara, 14). Au-delà de ce trafic " classique ", notons que les fouilles de Lattes ont livré des documents plus rares mais qui témoignent de l'ouverture du site vers un large marché : céramiques « celtiques" (Lattara, 14), perle égyptienne (Arnal et al., 1974), monnaies de Salapia et de Capoue, bronzes ptolémaïques, etc. La mise en lumière de la participation de Lattes, à partir du changement d'ère, à un commerce du vin en vrac, constitue une nouveauté importante.

Le dossier des contreparties indigènes reste toujours délicat à aborder et recouvrir l'ensemble des biens exportés sous le terme de biotos - produits bruts de première nécessité intéressant les Grecs, les Étrusques, puis les Romains facilite la chose. La place des céréales dans ces contreparties à partir de l'étude des structures de stockage a été mise en avant tant pour Lattes (Garcia, 1992) que pour le Languedoc méditerranéen (Garcia, 1987) et plus généralement pour la Méditerranée nord-occidentale (Garcia, 1997). O. Puertas a récemment tenté une spatialisation des activités agricoles et commerciales dans le delta du Lez à partir de l'analyse pollinique. Pour l'âge du Fer, il note notamment une intense 
exploitation agricole du terroir (Puertas, 1999, p. 46), ce qui avait déjà été mis en évidence par les études carpologiques et anthracologiques (Buxó, 1992 ; Ambert, Chabal, 1992). Mais le carottage réalisé à proximité du port met en avant un fort taux de Cerealia (25\%). En outre, le cortège de pollens de rudérales et messicoles associés est extrêmement restreint et peu diversifié. Selon les critères développés notamment par H. Richard, un tel spectre pollinique n'est pas représentatif d'un fait écologique particulier (mise en culture) mais traduit d'autres activités humaines (Richard, 1994). «La présence de sédiments argilo-organiques fins de type lagunaire et la localisation particulière du sondage dans la zone portuaire du site de Lattara permettent d'interpréter ces taux importants de Céréales comme représentatifs d'activités commerciales. Il n'est toutefois pas possible de déterminer le type d'activités (transport ou stockage) ni l'état d'élaboration des grains de Céréales » (Puertas, 1999, p. 47). Cette étude est à porter au dossier des activités commerciales, sans toutefois permettre d'apprécier l'évolution chronologique et quantitative de ce type de trafic.

La construction et/ou la réparation des embarcations est une activité souvent attestée dans les ports antiques où elle induit la présence de plages ou de cales en pente douce permettant de tirer les bateaux. Nos travaux de terrain n'ont rien apporté d'original dans ce domaine, sinon que les analyses archéoentomologiques entreprises récemment dans les sédiments de remplissage de plusieurs puits de Lattes ont mis en évidence la présence de Pselachus spadix, coléoptère vivant sur du bois flotté et qui pourrait indiquer l'existence d'un petit chantier naval (Lattara, 18) ; un phénomène identique avait été relevé à Marseille (Ponel, 2001). Il faut également garder en tête l'inscription de T. Eppilius Astrapton (Demougeot, 1966 ; Barruol, 1988), sur laquelle nous reviendrons et qui mentionne des fabr $(i)$, traditionnellement identifiés comme des charpentiers de marine.

Enfin, parmi les activités du port, la part de la pêche est naturellement à prendre en compte. À Lattes, dès les premières opérations archéologiques entreprises, l'abondance des artefacts liés à la pêche (hameçons, lests de filets) mis au jour par H. Prades (Arnal et al., 1974) et la présence remarquable de restes de poissons laissaient supposer le poids de cette activité dans la vie du port et l'économie de la cité. Les fouilles programmées depuis 1986 ont permis de confirmer cette impression. En développant l'étude des restes osseux de poissons, la pêche est apparue comme une activité importante pour la vie de la cité, d'abord vivrière (Sternberg, 1995 et 1999) et occasionnellement artisanale (Piquès, 2005 ; Garcia, Sternberg, à paraître).
On a déjà souligné l'absence de vestiges portuaires antérieurs au II ${ }^{\mathrm{e}}$ s. av. J.-C. À l'exception de Marseille, notre bilan bibliographique aboutissait à un constat similaire pour les autres sites préromains. On peut proposer que les caractéristiques topographiques des sites portuaires du littoral gaulois, et celles du site de Lattes en particulier, permettaient, moyennant des aménagements réduits (appontements...) et des pratiques simples (halage des embarcations sur les plages...), un développement raisonné des activités portuaires. Les produits étaient embarqués ou débarqués directement : le «port " était davantage un espace de manutention qu'un espace de stockage et de gestion. La structuration interne de la ville préromaine permettait ce type d'organisation : la voie 100, grande rue charretière orientée nord-sud, aboutissait à la porte ouvrant sur le port ; les quelques greniers mis au jour donnaient préférentiellement sur cette artère (Garcia, 1992). La diffusion, vers l'arrière-pays, des produits déchargés devait se faire par voie terrestre, ce que les volumes estimés (Py, 1999b, fig. 4) rendent parfaitement plausible. La ville apparaît davantage comme un centre de consommation, dont la localisation profite largement du commerce avec les Étrusques et les Grecs, que comme un centre de grande redistribution. Bien entendu, tout ceci doit être compris en termes de volume : l'intérêt des indigènes pour le vin devait être considérable et le statut de la ville ne devait pas pâtir (bien au contraire !) du volume, même réduit, des produits diffusés.

Ce n'est qu'à partir de la fin du III $^{\mathrm{e}} \mathrm{s}$. av. J.-C. que les importations de Marseille cèdent progressivement le pas à celles en provenance d'Italie. Les bateaux des $\mathrm{II}^{\mathrm{e}} \mathrm{s}$. et I ${ }^{\mathrm{er}} \mathrm{s}$. av. J.-C. sont en moyenne de plus fort tonnage que la plupart des embarcations des périodes archaïques et classiques (Tchernia, 1986 ; Pomey, 1997). Les premiers aménagements portuaires que nous avons dégagés (phase 1 : 175-125 av. J.-C.) peuvent être interprétés comme des constructions répondant, en particulier, à l'évolution du tonnage des navires. Les terrepleins situés devant la porte méridionale de la ville gagnent sur l'espace sublagunaire : ils permettent de se rapprocher des zones à plus fort tirant d'eau et facilitent ainsi les embarquements et les déchargements.

Les constructions (hangars ?) reconnues pour la phase 2 (125-25 av. J.-C.) traduisent elles aussi une évolution des pratiques portuaires. Elles peuvent avoir servi à protéger du matériel, voire des embarcations, ou bien à stocker provisoirement des marchandises. Le port s'affirme alors comme un espace autonome de gestion et de transit. Les marchandises ne gagnent plus directement la ville, peut-être même ne font-elles que transiter vers d'autres débarcadères. 
La diffusion des amphores italiques de type Dressel 1 en Languedoc revêt un caractère beaucoup plus intense que ne l'avait été celle des amphores étrusques ou grecques. Le trafic commercial des sites portuaires, dont celui de Lattes, connaît probablement un développement important. La ville consomme plus, mais elle redistribue plus encore ; elle acquiert sans doute un statut de port régional.

Dès 1974, H. Prades avait signalé la découverte en surface de la zone portuaire, en 1963, d'un chapiteau de style corinthien (Arnal et al., 1974, p. 22). Un fragment de chapiteau de même style a été retrouvé jeté dans un dolium du bâtiment 26/8, une base de pilastre corinthien a été placée en bordure du hangar à dolia et quatre bases de colonnes ont été mises au jour dans le bâtiment 34/20. Plusieurs fragments de fûts de colonnes et des tuiles en calcaire scié ont aussi été recueillis. Un autre entrepôt (zone 17) situé intra muros, en bordure de la rue 116, a également livré, en réemploi comme base, un chapiteau de style toscan (Py, Garcia, 1993, fig. 62). Tous ces éléments peuvent être datés des années 50-25 av. J.-C., peut-être un peu avant pour le chapiteau de style toscan si l'on le rapproche, comme le fait C. Landes (1995-1996), de celui, inscrit en gallo-grec, mis au jour à Substention (Castelnau-le-Lez, Hérault). Tous ces éléments plaident en faveur de l'existence d'une parure monumentale à Lattes au I ${ }^{\mathrm{er}}$ s. av. J.-C., d'une destruction brutale de ces bâtiments vers 20 av. J.-C. et de leur réemploi rapide lors de la mise en place de la grande terrasse portuaire. Il est évident qu'une telle rupture dans l'usage des ouvrages ne peut être liée qu'à des événements importants. C. Landes a proposé de rapprocher ces faits de la réorganisation de la Narbonnaise par Auguste entre 27 et 22 av. J.-C., qui pour le territoire des Arécomiques se manifesta par la perte d'autonomie de 24 oppida ignobilia au profit de Nîmes. Les blocs en réemploi mis au jour dans le port témoigneraient alors « d'un abandon et d'une spoliation progressifs des bâtiments publics peut-être devenus inutiles après la perte des libertés politiques au profit de Nîmes » (Landes, 1995-1996).

Pour le $\mathrm{I}^{\mathrm{er}} \mathrm{s}$. av. J.-C. l'étude des céramiques découvertes dans l'habitat montre " une baisse régulière du volume amphorique. Par rapport à la vaisselle consommée dans le même temps, en terme de fragments, le rapport reste ce qu'il était au $\mathrm{II}^{\mathrm{e}} \mathrm{s}$., dans les trois premiers quarts du $\mathrm{I}^{\mathrm{er}} \mathrm{s}$. $(0,5)$ mais il diminue dans le dernier quart $(0,37)$, rapport que l'on retrouve dans la seconde moitié du $\mathrm{I}^{\mathrm{er}} \mathrm{s}$. de n. è. » (Fiches, 1994, p. 370). Cet état de fait était interprété comme un « signe possible de la perte d'une certaine spécificité socio-économique de Lattes, de son importance en tant que complexe portuaire de premier rang » (Fiches, 1994, p. 348) : de « comptoir longtemps ouvert directement au commerce maritime, [Lattes est] devenu alors un port d'intérêt local »(Fiches, 1994, p. 371). Or, nous avons vu que l'état de la phase 3 (25 av. J.-C./75 apr. J.-C.) révèle un projet architectural cohérent dans lequel le bâtiment à dolia reflète la participation de Lattara à un trafic du vin en vrac qui touche alors toute la Méditerranée nord-occidentale. Cette découverte éclaire maintenant d'un jour nouveau l'étude de la céramique. La baisse du nombre d'amphores à partir de la fin du $\mathrm{I}^{\mathrm{er}} \mathrm{s}$. av. J.-C. est sans doute à mettre en rapport avec l'arrivée du vin en vrac et peut-être avec le développement d'une production locale (Buxó, 1992 ; Garcia, 1992 ; Py, Buxó, 2001). Le rôle commercial de Lattes - indépendamment de la perte de son autonomie politique s'est probablement accru à la période augustéenne. On peut imaginer que ce vin était diffusé dans la partie orientale de la cité de Nîmes, peut-être dans des amphores gauloises, comme plusieurs indices inédits nous le suggèrent. Ainsi, on comprend mieux pourquoi, alors que le nombre d'amphores baissait à Lattes, «en revanche, Lunel-Viel [échappait] à ce schéma en raison notamment d'une forte proportion d'amphores gauloises à pâte calcaire qui entraînent des rapports beaucoup plus élevés»(Fiches, 1994, p. 370). La diffusion des produits devait se faire par voie terrestre mais peut-être aussi par le fleuve et les étangs, conférant ainsi aux charpentiers navals et aux utriculaires une place essentielle dans la vie du vicus, comme en témoigne l'inscription d'Astrapton. Celle-ci, datée du $\mathrm{II}^{\mathrm{e}}$ s. apr. J.-C., fut découverte en 1965 en bordure septentrionale du domaine de Saint-Sauveur (Demougeot, 1966 ; Barruol, 1988) : «Deo Marti Aug(usto) / et Gen(io) col(legii) Sevir(orum) / Aug(ustalium), T(itus) Eppil(ius) Astrapton / fabr(i) et utric(larii) lattar(enses) / [ob ? pro ?] mer(ita ? itis ?) eor(um)». Elle fait état - comme nous l'avons déjà noté - de l'importance du collège des charpentiers navals mais aussi des utriculaires lattois. Ces derniers ont le plus souvent été interprétés comme des bateliers maniant des radeaux fabriqués avec des outres. Mais, en fait, un réexamen critique des différentes sources (Deman, 2002 ; Verdin, 2005) incite à désigner les utriculaires comme des fabricants d'outres destinées à la diffusion de denrées liquides vers l'intérieur des terres.

Les aménagements portuaires que nous avons mis au jour, au sud de la porte méridionale primitive, sont certainement associés à d'autres ouvrages. À la fin du I ${ }^{\text {er }}$ s. av. J.-C., en bordure orientale du site (zone 19), entre le parement externe du rempart et une berge d'un ancien bras du Lez, sont aménagées des petites cellules de stockage (dont 
l'état d'abandon a livré de nombreuses amphores de type Dressel 20). En ce lieu, un mur en petit appareil bien assisé dont les joints sont tracés au fer témoigne probablement d'une canalisation de ce bras du fleuve. Dans la zone 6, une rue large de $1,50 \mathrm{~m}$ à $2 \mathrm{~m}$ sépare de probables magasins également datés du I ${ }^{\mathrm{er}}$ s. apr. J.-C. Deux bâtiments importants sont également construits sur la place 23.

Comme nous l'avons proposé, les transformations architecturales radicales notées durant la phase 4 (75 apr. J.-C./225 apr. J.-C.) ne marquent peut-être pas des modifications dans les produits transportés et l'organisation générale du portus. Le terre-plein est certainement agrandi vers le sud, d'autres espaces portuaires ont peut-être été créés, la généralisation de l'usage des tonneaux (et des outres ?) a peut-être stoppé le commerce du vin en vrac sans que l'objet de ces échanges ne disparaisse.

L'abandon de Lattes est situé aux environs de 200 apr. J.-C. (Py, Garcia, 1993, p. 21) mais l'analyse du mobilier contenu dans les dolia du bâtiment 28/9 montre que certains bâtiments étaient encore en fonction à la fin du $\mathrm{III}^{\mathrm{e}}$ s. (phase 5 : 225 apr. J.-C./300 apr. J.-C.). De vicus portuaire, Lattara est peut-être alors devenue un simple débarcadère utilisé à des fins locales par les occupants des villae environnantes. 